Sotoudeh, M., Bongers-Loth, M. D., Roddatis, V., Čížek, J., Nowak, C., Wenderoth, M., Blöchl, P., Pundt, A. (2021): Hydrogen-related defects in titanium dioxide at the interface to palladium. - Physical Review Materials, 5, 12, 125801.

https://doi.org/10.1103/PhysRevMaterials.5.125801 


\title{
Hydrogen-related defects in titanium dioxide at the interface to palladium
}

\author{
Mohsen Sotoudeh $\odot,{ }^{1, *}$ Marian David Bongers-Loth, ${ }^{2}$ Vladimir Roddatis $\odot,{ }^{2,3}$ Jakub Č́žžek, ${ }^{4}$ Carsten Nowak, $, 2,5$ \\ Martin Wenderoth, ${ }^{6}$ Peter Blöchl $\odot,{ }^{7,8, \dagger}$ and Astrid Pundt $\odot^{2,9, \$}$ \\ ${ }^{1}$ Institute of Theoretical Chemistry, Ulm University, Albert-Einstein-Allee 11, 89081 Ulm, Germany \\ ${ }^{2}$ Institut für Materialphysik, Universität Göttingen, Friedrich-Hund-Platz 1, 37077 Göttingen, Germany \\ ${ }^{3}$ Helmholtz Centre Potsdam, GFZ German Research Centre for Geosciences Telegrafenberg, 14473 Potsdam, Germany \\ ${ }^{4}$ Department of Low-temperature Physics, Charles University in Prague, V Holešovičkách 2, 18000 Praha 8, Czech Republic \\ ${ }^{5} X L A B$, Universität Göttingen, Justus-von-Liebig-Weg 8, 37077 Göttingen, Germany \\ ${ }^{6} I V$. Physikalischen Institut, Universität Göttingen, Friedrich-Hund-Platz 1, 37077 Göttingen, Germany \\ ${ }^{7}$ Institute for Theoretical Physics, Clausthal University of Technology, Leibnizstr. 10, 38678 Clausthal-Zellerfeld, Germany \\ ${ }^{8}$ Institut für Theoretische Physik, Universität Göttingen, Friedrich-Hund-Platz 1, 37077 Göttingen, Germany \\ ${ }^{9}$ Institut für Angewandte Materialien-Werkstoffkunde, Karlsruher Institut für Technologie, Kaiserstr. 12, 76131 Karlsruhe, Germany
}

(Received 21 June 2021; revised 8 November 2021; accepted 24 November 2021; published 10 December 2021)

\begin{abstract}
A metal oxide support and a catalytically active metal are the two main ingredients for complex catalysts used in heterogeneous catalysis. The gas environment can change the catalyst during the reaction, modifying its structural and electronic properties. Here, we use monochromated electron energy loss spectroscopy (EELS) to reveal hydrogen-pressure-dependent changes of the electronic structure at the $\mathrm{Pd} / \mathrm{rutile}-\mathrm{TiO}_{2}$ interface in an environmental transmission electron microscope (ETEM). Hydrogen-induced changes are observed in rutile$\mathrm{TiO}_{2}$ within $2 \mathrm{~nm}$ from the interface at $10 \mathrm{~Pa}$ of hydrogen pressure, in the Ti $L_{3,2}$ EEL spectra. Lower pressures such as $1 \mathrm{~Pa}$ show no changes in the EEL spectra. We attribute the observed changes in the EEL spectra to hydrogen-induced defects accumulating in the vicinity of the interface. Based on DFT calculations, we developed a thermodynamic multistate defect (TMD) model of the interface and the bulk of the rutile- $\mathrm{TiO}_{2}$. This TMD model predicts high concentrations of positively charged defects accumulating at the interface. The presence of the Schottky barrier stabilizes these defects by significantly lowering their formation energy. Our findings provide important insights into catalytic processes taking place at metal/metal oxide interfaces in hydrogen gas environments.
\end{abstract}

DOI: 10.1103/PhysRevMaterials.5.125801

\section{INTRODUCTION}

Metal/oxide systems using titanium dioxide $\left(\mathrm{TiO}_{2}\right)$ as a support have been used in a broad range of technological applications where they serve as model systems for fundamental process studies in surface science [1,2], catalysis [3-16], the field of resistive switching [17-19], as well as for Schottky contacts [20-23]. One central question in these studies is how chemical and physical properties of the metal/ $/ \mathrm{TiO}_{2}$ interface are influenced by an electric field [19], by metal-support interaction [11], or by defect concentrations [21,22]. The latter can be also influenced by the environment, e.g., by the presence of hydrogen, as utilized in for catalysts and gas sensors.

Defects, such as oxygen vacancies, Ti vacancies, and $\mathrm{Ti}$ interstitials, and their properties in bulk rutile- $\mathrm{TiO}_{2}\left(\mathrm{r}-\mathrm{TiO}_{2}\right)$ have been experimentally studied [1,6,24-32]. On the other side, the hydrogen concentration in pristine $\mathrm{r}-\mathrm{TiO}_{2}$ crystals can be found [33] between $10^{17} \mathrm{~cm}^{-3}$ and $10^{19} \mathrm{~cm}^{-3}$. Interstitial hydrogen $[34,35]$ and hydrogenated oxygen vacancy

\footnotetext{
*mohsen.sotoudeh@uni-ulm.de

†peter.bloechl@tu-clausthal.de

†astrid.pundt@kit.edu
}

[35] $\left(\mathrm{H}_{\mathrm{O}}\right)$ are detected by infrared spectroscopy [36] and discussed as two important hydrogen-related defects, which act as dopants in $\mathrm{r}-\mathrm{TiO}_{2}$.

According to recent theoretical studies on bulk $\mathrm{TiO}_{2}$ [35,37-41], the favorable adsorption sites for $\mathrm{H}$ atoms are oxygen vacancies $\left(\mathrm{V}_{\mathrm{O}}\right)$, and, in the absence of vacancies, interstitial sites $\left(\mathrm{H}_{\mathrm{i}}\right)$. All these point defect studies were carried out using first-principles calculations based on density-functional theory (DFT).

Palladium (Pd) or platinum (Pt) are often used as metal partners. They are highly active in hydrogenation reactions [42], and for dissociative chemisorption of $\mathrm{H}_{2}$ molecules at their surfaces [43-45]. Pd is a well-studied catalyst and also a system for hydrogen ad- and absorption [46-48]. Hydrogen solves on interstitial sites in the Pd lattice in the Pd-H $\alpha$ phase and the Pd-H hydride phase [43,49]. The solubility of $\mathrm{H}$ depends on the hydrogen chemical potential [50]. It is, for thin films, also affected by the film's microstructure [51-54] and the mechanical stress state [55]. For low chemical potentials, the hydrogen-related mechanical stress is small [56]. For high chemical potentials of $\mathrm{H}$, the mechanical stress can be in the GPa-range [52]. The room-temperature kinetics of $\mathrm{H}$ in $\mathrm{Pd}$ is fast [57]. The $\mathrm{H}$ diffusion coefficient of $3.2 \times 10^{-11} \mathrm{~m}^{2} / \mathrm{s}$ leads to diffusion over $\mu \mathrm{m}$ distances within seconds. For a Pd 
thin film, this allows for fast equilibration and, further, to fast transport of atomic hydrogen to the film/ $/ \mathrm{TiO}_{2}$ interface.

At the $\mathrm{Pd} / \mathrm{TiO}_{2}$ interface, the energetic difference between the work function of $\mathrm{Pd}$ and the electron affinity of $\mathrm{TiO}_{2}$ results in electron transfer. A space charge layer builds up and a Schottky barrier establishes at the contact $[58,59]$ according to Schottky's classical picture. Hydrogen diffuses through the $\mathrm{Pd}$ to the interface where it spills over into the $\mathrm{TiO}_{2}$ and affects the Schottky barrier thickness and/or height [21]. Irreversible changes in the conductivity of hydrogen loaded $\mathrm{Pd} / \mathrm{TiO}_{2}$ hint on the presence of stable hydrogen-oxygen vacancy complexes [22]. Hydrogen additionally lowers the critical field necessary for electroforming processes and can eventually lead to the formation of substoichiometric phases in the titanium dioxide [22]. Recent experiments reveal additional local (spatial) changes of the Schottky barrier height that could be attributed to the formation of substoichiometric Magnéli phases $\left(\mathrm{Ti}_{n} \mathrm{O}_{2 n-1}, n=4\right.$ to 10$)$ in the vicinity of the interface [22]. All suggested interpretations [22,60] of the observed Schottky barrier height changes hint on a dominant contribution of defects in the near-interface region in the $\mathrm{TiO}_{2}$. The microscopic details of the interface changes are still under debate. However, a strong contribution of defects is beyond question. The dominant type of hydrogen-related defects in $\mathrm{r}-\mathrm{TiO}_{2}$ is still unknown, especially in the close vicinity to the Pd interface.

In this paper, we determine hydrogen-related changes in $\mathrm{r}-\mathrm{TiO}_{2}$ in the nanometer vicinity of the $\mathrm{Pd} / \mathrm{r}-\mathrm{TiO}_{2}$ interface. Experimentally, we use electron energy-loss spectroscopy (EELS) in an environmental TEM (ETEM). First-principle calculations based on the generalized gradient approximation (GGA) of the DFT were carried out to assess the influences of different defects on the electronic density of states (DoS). Results originating from these calculations serve as input parameters for a thermodynamic multistate defect model (TMD model). This TMD model allows to implement the experimental conditions and to gain information about defect concentrations in the $\mathrm{r}-\mathrm{TiO}_{2}$ and at the interface to the metal.

\section{METHODS}

\section{A. $\mathrm{Pd} / \mathrm{TiO}_{2}$ sample preparation and characterization}

$\mathrm{Pd}(111)$ films were deposited on pretreated $\mathrm{r}-\mathrm{TiO}_{2}(110)$ crystals (CrysTec $\mathrm{GmbH}$, Berlin, epi-polished), by magnetron sputter deposition in a high vacuum system. The $\mathrm{r}-\mathrm{TiO}_{2}$ substrates were etched in $20 \%$ fluoric acid, rinsed in purified water and annealed for $1 \mathrm{~h}$ at $1173 \mathrm{~K}$ in an $\mathrm{O}_{2}$ gas stream of approximately $10^{5} \mathrm{~Pa}$. Such pretreatment results in the formation of clean and smooth well-defined surface terraces on $\mathrm{r}-\mathrm{TiO}_{2}$ as verified by $\mathrm{x}$-ray photoelectron spectroscopy (XPS) and atomic force microscopy (AFM). Subsequently, the Pd films were deposited at $1024 \mathrm{~K}$ in an oxygen atmosphere with a pressure of $7 \times 10^{-3} \mathrm{~Pa}$ and a sputter rate of approximately $11.8 \mathrm{~nm} / \mathrm{min}$. After film deposition, the samples were cooled down in the oxygen atmosphere to about $373 \mathrm{~K}$. The chosen deposition conditions resulted in Pd grain sizes above $1 \mu \mathrm{m}$. Further details are provided elsewhere [61].

Cross-sectional specimens required for high-resolution transmission electron microscopy (HRTEM) and EELS stud- ies are prepared by mechanical polishing and subsequent low-angle $\left(6^{\circ}\right) \mathrm{Ar}^{+}$ion milling at $3 \mathrm{kV}$ in PIPS 691 (Gatan). At the final stage of ion milling, the acceleration voltage was gradually decreased to $0.5 \mathrm{kV}$ according to the procedure described by Süess et al. [62].

Variable energy positron annihilation spectroscopy (VEPAS) studies on $\mathrm{Pd} / \mathrm{r}-\mathrm{TiO}_{2}$ reveal no detectable change of the Ti-vacancy concentration in $\mathrm{r}-\mathrm{TiO}_{2}$ in the here-addressed pressure range. VEPAS probes for vacancy concentrations between $10^{-7}$ to $10^{-4}$ per atom [63]. Further, the concentration of the related $\mathrm{Ti}$ interstitials is, here, regarded as low, with respect to the concentration of oxygen vacancies, under the chosen preparation conditions [31] and assumed to be constant in the experimentally studied pressure range. Therefore, we do not consider any Ti-related defects in our simulations. Details on the VEPAS measurements are presented in Appendix A.

\section{B. Electron energy-loss spectroscopy (EELS) experimental details}

The experiments on the $\mathrm{Pd} / \mathrm{TiO}_{2}$ interfaces were performed using an FEI Titan 80-300 ETEM operated at $300 \mathrm{keV}$. The ETEM is equipped with a Cs-image corrector, monochromator and GATAN image filter (GIF) Quantum 965. TEM experiments were performed in a high vacuum $\left(10^{-5} \mathrm{~Pa}\right)$ and at $\mathrm{H}_{2}$ gas pressures up to $10 \mathrm{~Pa}$. The sample was equilibrated in $\mathrm{H}_{2}$ for 1 hour at each pressure. During this time the electron beam in the ETEM was blanked. The hydrogen loaded Pd can be regarded as an atomic hydrogen source for this study. Spectrum images (SI) were collected at different places in the close nanometer-range vicinity of the $\mathrm{Pd} / \mathrm{TiO}_{2}$ interface, at selected $\mathrm{H}_{2}$ gas pressure ranging from $10^{-1} \mathrm{~Pa}$ to $10 \mathrm{~Pa}$. To reveal changes in the Ti $L$ electron energy-loss near-edge structure (ELNES) of about $100 \mathrm{meV}$ an advanced procedure was used that is described in details in Appendix B.

\section{Computational details}

First-principles calculations based on density-functional theory (DFT) $[64,65]$ were performed on pristine $\mathrm{r}-\mathrm{TiO}_{2}$ and $\mathrm{r}-\mathrm{TiO}_{2}$ containing selected defects. We used the generalized gradient approximation (GGA) functional PBE [66].

The calculations were carried out with the projector augmented wave (PAW) [67] method using the CP-PAW code [68]. The augmentation has been set up using the systematic projector construction [69]. For the augmentation, a $s^{2} p^{2} d^{1}$ $(3 s, 3 p, 3 d, 4 s$, and $4 p$ orbitals) set of projector functions was used for the Ti atom, and a $s^{2} p^{1} d^{1}(2 s, 2 p, 3 s$, and $3 d$ orbitals) set for the $\mathrm{O}$ atom. The superscripts denote the number of projector functions per angular momentum channel. For the Ti atom, the $3 s$ and $3 p$ core electrons were treated on the same level as the valence electrons.

A plane wave cutoff of 40 Ry has been chosen for the wave functions and $80 \mathrm{Ry}$ for the charge density. The Brillouin-zone integration has been performed with the linear tetrahedron method [70,71] and the so-called Blöchl corrections [72]. A $5 \times 5 \times 8$ k-point mesh has been used for the unit cell containing two formula units. Defect calculations were simulated by $2 \times 2 \times 3$ supercells with 72 atoms plus the defect. All atomic 

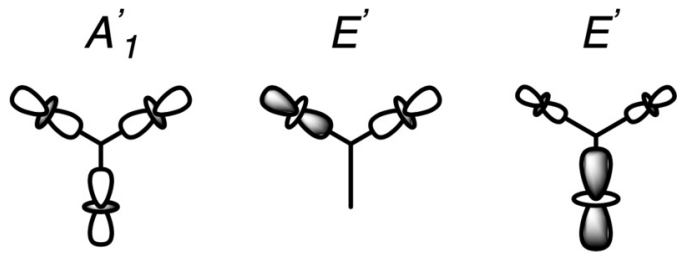

FIG. 1. Corresponding Ti- $d$ orbitals representing the local symmetry at an O-site (defect center), in the (110) plane. The Ti- $d$ states pointing towards the defect center are dominated by the approximate $\mathrm{D}_{3 \mathrm{~h}}$ symmetry of the defect. They are classified as $A_{1}^{\prime}$ and $E^{\prime} . E^{\prime}$ appears in two degenerate modifications. For the other $d$ orbitals on the Ti neighbors of the defect, we refer to the approximate octahedral symmetry $\left(\mathrm{O}_{\mathrm{h}}\right)$.

positions are optimized without symmetry constraints. A theoretical lattice constant $4.562 \AA$ of bulk r- $\mathrm{TiO}_{2}$ was obtained, in good agreement with the experimental [73] value of $4.593 \AA$. The calculated $c / a$ ratio matches the experimental [73] ratio of 0.644. This lattice constant was used for the supercells. Collinear spin polarization was allowed in all calculations.

Three different defect types in $\mathrm{r}-\mathrm{TiO}_{2}$ were selected: The oxygen vacancy $\mathrm{V}_{\mathrm{O}}$, the interstitial hydrogen $\mathrm{H}_{\mathrm{i}}$, and the oxygen vacancy filled with hydrogen $\mathrm{H}_{\mathrm{O}}$. The interstitial titanium $\mathrm{Ti}_{\mathrm{i}}$ was not considered as the concentration was found experimentally not to depend on the hydrogen partial pressure (Appendix A).

Crystal field splitting divides the Ti- $d$ orbitals, located at the center of a regular $\mathrm{TiO}_{6}$ octahedron, into high-lying, doubly degenerate $e_{g}$ and low-lying triply degenerate $t_{2 g}$ orbitals. Note that the crystal-field splitting originates from the covalent interaction with the oxygen neighbors. The stronger $\sigma$ bond of the $e_{g}$ states in comparison to the $\pi$ bonds formed by the $t_{2 g}$ states, shifts the antibonding $e_{g}$ states energetically above the $t_{2 g}$ states.

The molecular orbitals associated with defect electronic states are related to the surrounding Ti- $d$ orbitals. Linear combinations of these orbitals result in bonding and antibonding states. The $e_{g}$ orbitals on the Ti neighbors of the defect can be divided into two situations: The situation where the orbitals point towards the defect center. They can be superimposed into one $A_{1}^{\prime}$ orbital and two $E^{\prime}$ orbitals, as shown in Fig. 1. In the second situation, the orbitals point orthogonal to this direction. It is denoted as $\delta e_{g}$. This situation appears not to be affected by the presence of defects, as will be shown later.

Furthermore, we considered the Ti- $t_{2 g}$, which are classified according to the approximate octahedral symmetry of the $\mathrm{Ti}$ site.

In order to represent the Ti- $d$ states pointing towards the defect center, the local $z$ axis for the Ti- $d$ orbitals has been defined by the Ti-O bond pointing away from the defect center. A second, approximately orthogonal Ti-O bond has been used to define the $x z$ plane.

The formation energy of defect $X_{\sigma}$ is given by the defect energy $E_{\sigma}$ and the chemical potentials for electrons, oxygen and hydrogen atoms:

$$
E_{f}\left[X_{\sigma}\right]=E_{\sigma}-\eta_{O, \sigma} \mu_{O}-\eta_{H, \sigma} \mu_{H}-\eta_{e, \sigma} \mu_{e} .
$$

TABLE I. $d$ orbitals and their symmetry label in the setting of the ideal octahedral $\left(\mathrm{O}_{\mathrm{h}}\right)$ and the orthorhombic distorted octahedral $\left(\mathrm{D}_{2 \mathrm{~h}}\right)$ symmetry group of $\mathrm{r}-\mathrm{TiO}_{2}$. The orbitals refer to the local coordinate system with the $z$ axis along the orthorhombic distortion. The corresponding white lines $\left(a, b, b^{\prime}, c\right.$, and $\left.d\right)$ occurring in EEL spectra of the Ti $L_{3,2}$ edge are given and associated with the energy states.

\begin{tabular}{lcccc}
\hline \hline orbital & $\begin{array}{c}\text { Symmetry } \\
\text { label in } \mathrm{O}_{\mathrm{h}}\end{array}$ & $\begin{array}{c}\text { Symmetry } \\
\text { label in } \mathrm{D}_{2 \mathrm{~h}}\end{array}$ & $\begin{array}{c}L_{3} \text { white } \\
\text { line }\end{array}$ & $\begin{array}{c}L_{2} \text { white } \\
\text { line }\end{array}$ \\
\hline$d_{x z}$ & $t_{2 g}$ & $b_{2 g}$ & $a$ & $c$ \\
$d_{y z}$ & $t_{2 g}$ & $a_{g}$ & $a$ & $c$ \\
$d_{x y}$ & $t_{2 g}$ & $b_{3 g}$ & $a$ & $c$ \\
$d_{3 z^{2}-r^{2}}$ & $e_{g}$ & $a_{g}$ & $b$ & $d$ \\
$d_{x^{2}-y^{2}}$ & $e_{g}$ & $b_{1 g}$ & $b^{\prime}$ & $d$ \\
\hline \hline
\end{tabular}

The defect energies $E_{\sigma}$ and stoichiometric factors $\eta_{O, \sigma}, \eta_{O, \sigma}$, and $\eta_{H, \sigma}$ are given below in Table III. The defect energy $E_{\sigma}$ is calculated as the energy difference $E\left[\mathrm{TiO}_{2}: X_{\sigma}\right]-E\left[\mathrm{TiO}_{2}\right]$ of the supercell with and without the defect. The lattice constants of the supercell have been taken from the calculations of bulk r-TiO ${ }_{2}$, provided in Table II.

In order to avoid large values without physical significance, we introduce reference values for each of the three chemical potentials. We choose the valence band top $\mu_{e}^{\text {ref }}=\epsilon_{v}$ for the electron chemical potential and one-half of the calculated molecular energies $\mu_{O}^{\mathrm{ref}}=\frac{1}{2} E\left[\mathrm{O}_{2}\right]$ and $\mu_{H}^{\mathrm{ref}}=\frac{1}{2} E\left[\mathrm{H}_{2}\right]$ for oxygen and hydrogen atoms, respectively.

In our supercell, the defects in $\mathrm{r}-\mathrm{TiO}_{2}$ produce a deviation of the valence-band maximum and conduction-band minimum ranging between $+0.15 \mathrm{eV}$ and $-0.02 \mathrm{eV}$ from the calculated bulk values. In our calculations, we measure the electron energies relative to the valence-band top of the perfect $\mathrm{r}-\mathrm{TiO}_{2}$ crystal.

The thermodynamic charge state level

$$
\epsilon\left(q_{1} / q_{2}\right)=\frac{E\left[X^{q_{2}}\right]-E\left[X^{q_{1}}\right]}{q_{2}-q_{1}}
$$

defines the position of the electron chemical potential $\mu_{e}$ for which two charge states $\left(q_{1}\right.$ and $\left.q_{2}\right)$ coexist in the same quantity. For lower $\mu_{e}$ the more positive charge state is thermodynamically stable and for higher $\mu_{e}$ the more negative charge state is thermodynamically stable. $\mu_{e}$ is commonly referred to as the Fermi level.

TABLE II. Lattice parameters $a$ and $c$, internal parameter $u$ [73] and band gaps of $\mathrm{r}-\mathrm{TiO}_{2}$ from the PBE method, and experiments $[73,78,79]$.

\begin{tabular}{lcc}
\hline \hline Quantity & PBE & Experiment \\
\hline$a(\AA)$ & 4.562 & 4.593 \\
$c / a$ & 0.644 & 0.644 \\
$u / a$ & 0.302 & 0.305 \\
Band gap $(\mathrm{eV})$ & 1.905 & 3.05 \\
Heat of formation $(\mathrm{eV})$ & -8.56 & -9.74 \\
\hline \hline
\end{tabular}




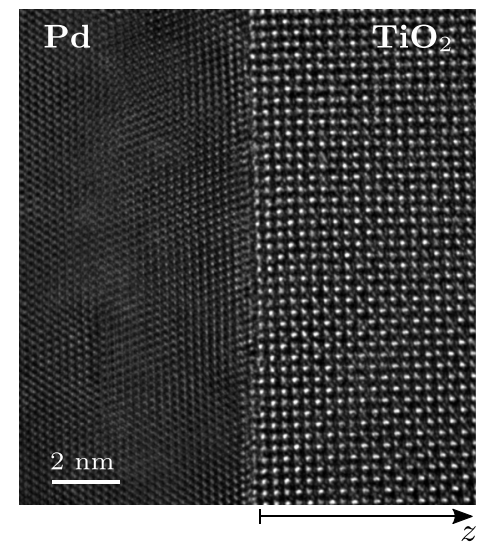

(a)

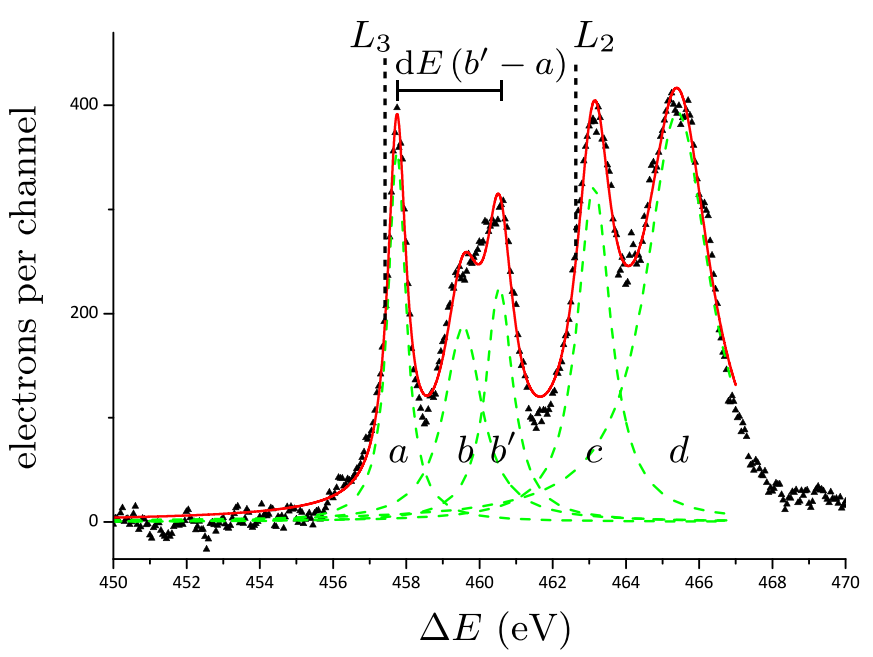

(b)

FIG. 2. (a) HRTEM picture of an as prepared $\mathrm{Pd} / \mathrm{r}-\mathrm{TiO}_{2}$ interface. A sharp interface between one to two monolayers thickness is revealed and no one or higher dimensional defects can be found in the interface region. (b) Typical Ti $L_{3,2}$ electron loss near edge structure (ELNES) (triangles) after a Hartree-Slater background (HSB) correction. Fitting with five Lorentz functions (green-dashed lines) yields an envelope function (red line), which describes the features in the Ti $L_{3,2}$ ELNES appropriately well. Indicated are the four white lines $\left(a, b / b^{\prime}, c, d\right)$ as well as the energy splitting $\mathrm{d} E\left(b^{\prime}-a\right) . \Delta E$ is the measured energy loss. This spectrum was detected in the as prepared state, for all distances.

\section{RESULTS}

\section{A. EELS experiments}

Figure 2 shows a HRTEM-image confirming a flat and sharp interface between the $\mathrm{Pd}$ film and the $\mathrm{r}-\mathrm{TiO}_{2}$ substrate. EEL spectra were collected in $\mathrm{r}-\mathrm{TiO}_{2}$ in the $0-20 \mathrm{~nm}$ vicinity to the interface at different $\mathrm{H}_{2}$ gas pressure below $1 \mathrm{~Pa}$. Details about the spectra evaluation are given in Appendix B, Fig. 10. For different $\mathrm{H}_{2}$ pressures below $1 \mathrm{~Pa}$, all EEL spectra show similar results, reflecting the bulk spectrum of $\mathrm{r}-\mathrm{TiO}_{2}$ shown in Fig. 2(b). Figure 2(b) focuses on the $\mathrm{Ti}$ $L$ edge. Any valence change or local symmetry change due to the presence of defects leads to modifications of the sys- tem's energy levels, which is especially measurable in EELS on the Ti $L$ edge $[18,74]$. The Ti $L_{3,2}$ edge originates from excitation of Ti- $2 p$ state electrons into the unoccupied states of the conduction band. As the Ti- $2 p$ states are split due to spin-orbit coupling $\left(2 p_{3 / 2}\right.$ and $2 p_{1 / 2}$ state) the two related $L_{3}$ and $L_{2}$ edges appear [74], as denoted in Fig. 2(b). They can be fitted with five Lorentz functions [green-dashed lines in Fig. 2(b)] corresponding to different orbitals (relations are given in Table I) yielding an envelope function [red line in Fig. 2(b)].

Remarkable differences of the Ti $L$ edge spectrum are observed at a hydrogen gas pressure of $10 \mathrm{~Pa}$, in the vicinity of the interface. Three representative spectra are shown in Figs. 3(a) and 3(b), for different distances from the interface. The Ti $L$ edge spectrum obtained at $1.5 \mathrm{~nm}$ distance from the interface shows distinct differences compared to the bulk spectrum, namely the shift by about $150 \mathrm{meV}$ in the $b, b^{\prime}$, and $d$ lines region. These differences are shown in Fig. 4(b). At a distance of $4.5 \mathrm{~nm}$ no differences are abserved with respect to the conventional bulk spectrum of $\mathrm{r}-\mathrm{TiO}_{2}$. Thus, for distances $\geqslant 4.5 \mathrm{~nm}$ the spectra resemble that of the bulk $\mathrm{r}-\mathrm{TiO}_{2}$. We interpret "extra" features in the spectra close to the interface as noise due to the decreasing intensity of the EELS signal. Fitting the ENLES with the five Lorentz functions is not influenced by this noise. Therefore analysis of the individual peak positions gives, inter alia, the energy splitting $\mathrm{d} E\left(b^{\prime}-a\right)$. It should be noted that the method was tested on interfaces before hydrogen loading. No changes in the Ti $L$ ELNES could be found here, as function of the interface distance.

The energy splitting $\mathrm{d} E\left(b^{\prime}-a\right)$ is shown in Fig. 4 as a function of the distance from the interface $z$, for hydrogen partial pressures of $1 \mathrm{~Pa}$ and $10 \mathrm{~Pa}$. At $1 \mathrm{~Pa} \mathrm{H}_{2}, \mathrm{~d} E\left(b^{\prime}-a\right)$ is about $2.8 \mathrm{eV}$ and does not depend on $z$, thereby resembling the bulk value [75]. At an $\mathrm{H}_{2}$ partial pressure of $10 \mathrm{~Pa}, \mathrm{~d} E\left(b^{\prime}-a\right)$ decreases by about $150 \mathrm{meV}$ when approaching the interface. This effect sets in below $2 \mathrm{~nm}$ from the interface.

The difference spectrum between the near interface $(1.5 \mathrm{~nm})$ spectrum and the bulk $(4.5 \mathrm{~nm})$ spectrum allows visualizing changes in the DoS and the related orbitals. These difference spectra are shown in Figs. 3(a) and 3(b) below the corresponding EEL spectra. In the difference spectrum of the $L_{3}$ edge shown in Fig. 3(a), states disappear at about $458 \mathrm{eV}$ and $461 \mathrm{eV}$ (gray-shaded area with $\mathrm{I} / \mathrm{I}_{0}<0$ ), while new states appear between $458.35 \mathrm{eV}$ and $460.55 \mathrm{eV}$ (gray-shaded area with $\mathrm{I} / \mathrm{I}_{0}>0$ ). This indicates that some energy states are shifted by about $1 \mathrm{eV}$ to $2 \mathrm{eV}$ with respect to the EELS in the bulk $\mathrm{r}-\mathrm{TiO}_{2}$. Below about $457.5 \mathrm{eV}$ the difference signal reflects the data scatter. The difference spectrum of the $L_{2}$ edge shown in the lower part of Fig. 3(b) shows similar results: States disappear at about $463.4 \mathrm{eV}$ and $466.4 \mathrm{eV}$, while new states appear between $463.8 \mathrm{eV}$ and $465.55 \mathrm{eV}$. The effect in the difference spectra is stronger for the $L_{3}$ edge than for the $L_{2}$ edge. We relate this to the stronger lifetime related broadening in the $L_{2}$ edge $[75,76]$.

Interestingly, the observed small shift of the $b^{\prime}$ peak by about $150 \mathrm{meV}$ [Fig. 4(b)] is the result of a chemically induced shift of states in the order of $1 \mathrm{eV}$. If this chemically induced shift occurs only on a fraction of $\mathrm{Ti}$ sites in the $\mathrm{r}-\mathrm{TiO}_{2}$ lattice, namely at $\mathrm{Ti}$ sites that are affected by a defect, the total EEL spectra can show comparably small shifts. Our 

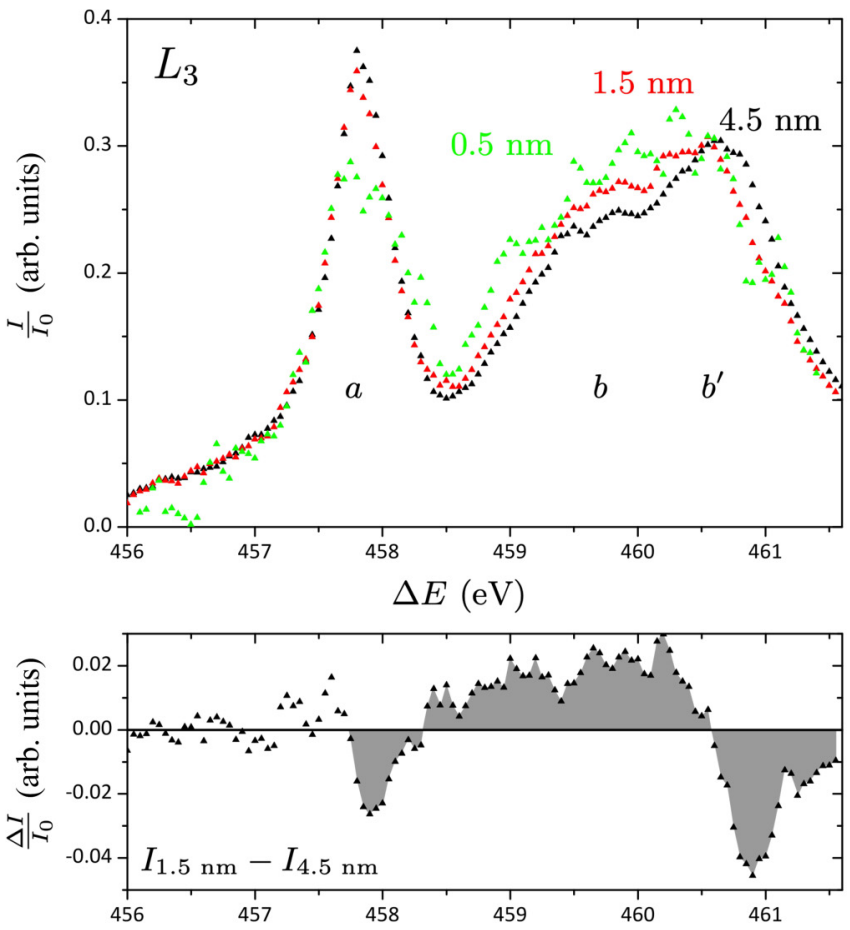

(a)
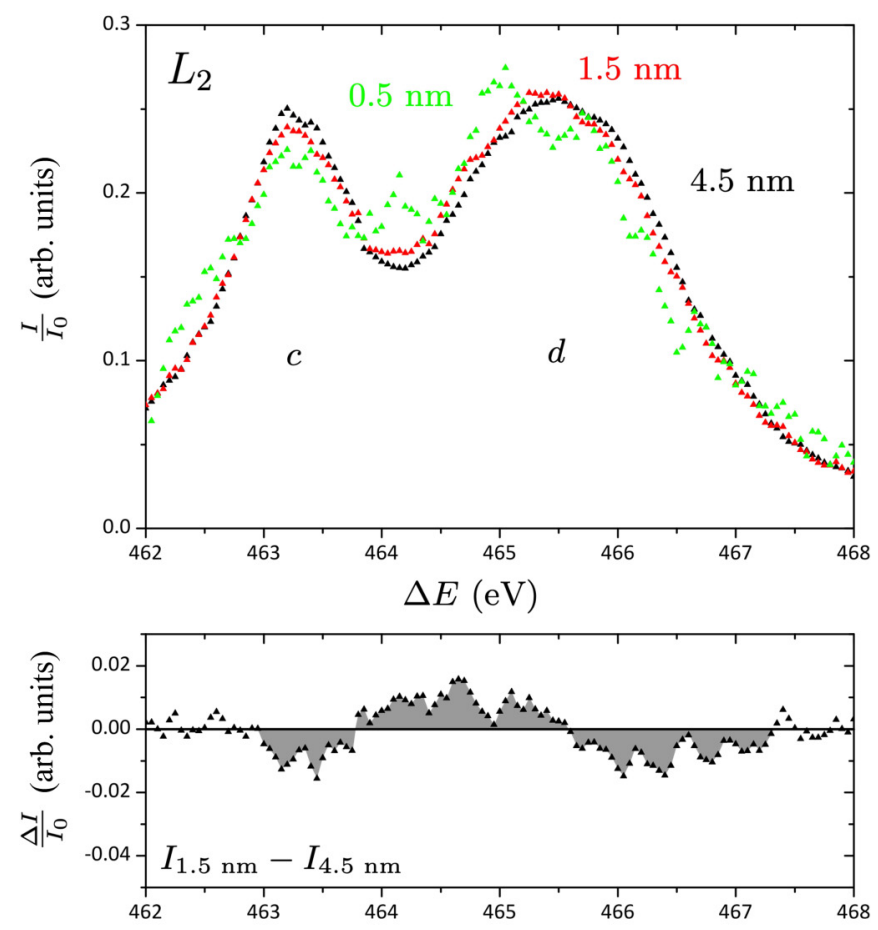

(b)

FIG. 3. Ti $L$ ELNES shown for different distances to the interface, at a hydrogen partial pressures of $10 \mathrm{~Pa}$ : (a) the $\mathrm{Ti} L_{3}$ edge and (b) the Ti $L_{2}$ edge spectrum in the $2 \mathrm{~nm}$ vicinity of the interface. All spectra are normalized to the area of the respective edge $\mathrm{I}_{0}$. The normed intensity difference $\Delta \mathrm{I} / \mathrm{I}_{0}$ between the $1.5 \mathrm{~nm}$ spectrum and the $4.5 \mathrm{~nm}$ spectrum is plotted in the lower part of each graph. The difference spectra visualize a shift of states (gray-shaded area) in the corresponding Ti $L$ edge.

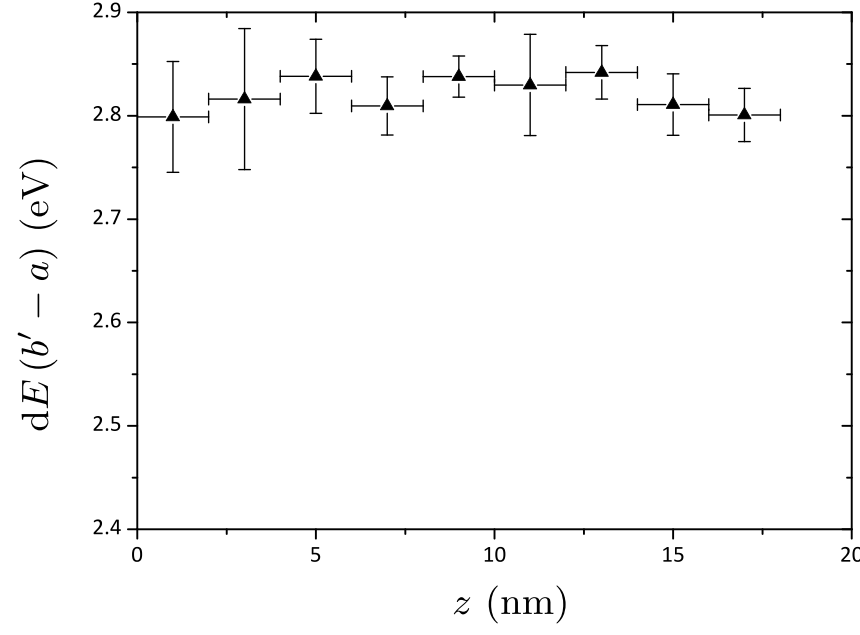

(a)

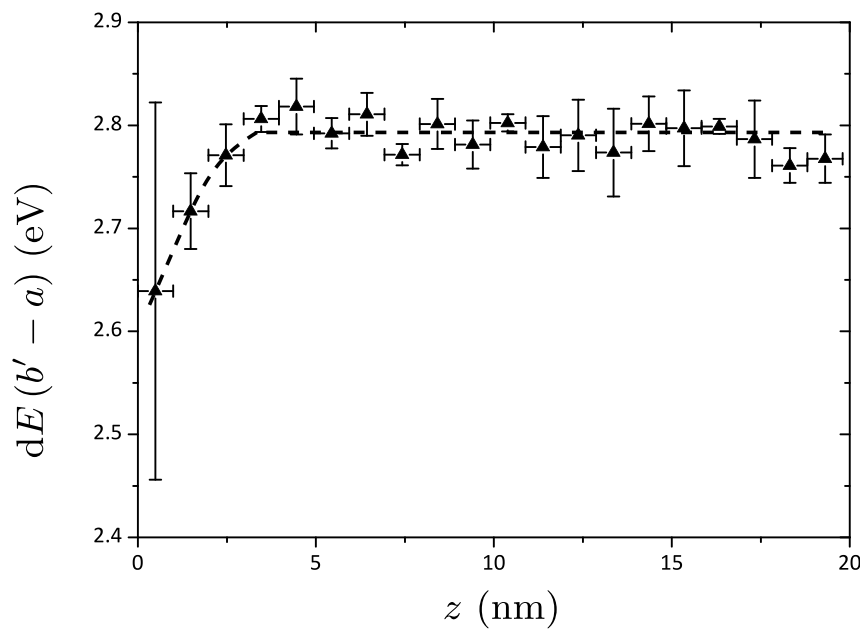

(b)

FIG. 4. Analysis of Ti $L$ ELNES on $\mathrm{Pd} / \mathrm{TiO}_{2}$, as function of the interface distance $z$, for the two selected hydrogen partial pressures (a) $1 \mathrm{~Pa}$ and (b) $10 \mathrm{~Pa}$. Energy splitting $\mathrm{d} E\left(b^{\prime}-a\right)$ between the $b^{\prime}$ and $a$ white lines are shown. (a) an constant energy difference of about $2.8 \mathrm{eV}$ is measured. (b) at $10 \mathrm{~Pa} \mathrm{H}_{2}$ the energy splitting decreases near the interface, starting at about $2 \mathrm{~nm}$ distance. The black-dashed line is plotted to guide the eye.

hypothesis here is that defects are located in $\mathrm{r}-\mathrm{TiO}_{2}$ in the close vicinity of the interface of the Pd metal. These defects are induced by the presence of hydrogen. The shift of the $b^{\prime}$ peak position, consequently, contains information about the local defect concentration. The corresponding defect concentrations are addressed in Sec. III C.

\section{B. Simulations}

In order to shed light on the experimental findings we performed calculations on hydrogen and oxygen-vacancy related defects. The nature of the defects is investigated using the projected density of states, wave function plots and formation energies. 


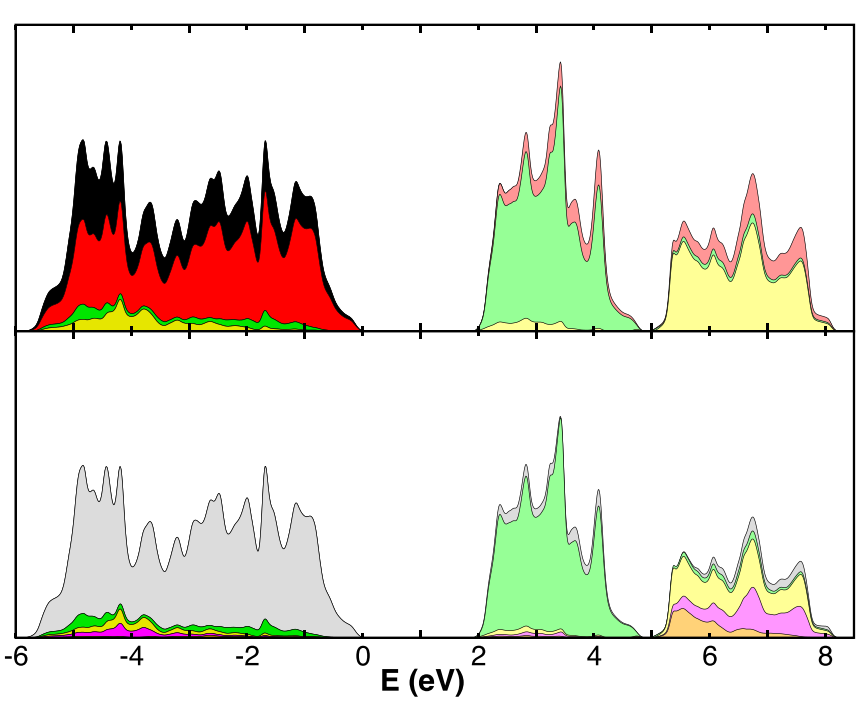

FIG. 5. Density of states (DoS) for $\mathrm{r}-\mathrm{TiO}_{2}$. The total DoS is given in black and gray, in the top and bottom graph respectively. The projected DoS are shown in red for oxygen, in green for $t_{2 g}$-like and yellow for $e_{g}$-like Ti- $d$ orbitals. Unoccupied states are drawn in a lighter color than the filled states. The energy zero is set to the top of the valence band. The bottom graph treats the perfect oxygen site as a defect with symmetry adapted orbitals. Hence, the $A_{1}^{\prime}$ (orange), $E^{\prime}$ (magenta), $\delta-e_{g}$ (yellow), and $\delta-t_{2 g}$ (green) orbitals are shown as the sum of the corresponding three Ti neighbors of the oxygen site. These symmetry adapted orbitals are defined in Sec. II C.

The calculated DoS of $\mathrm{r}-\mathrm{TiO}_{2}$ is shown in Fig. 5. The filled valence band, which extends from -6 to $0 \mathrm{eV}$, is predominantly of O- $p$ character (red) with some contribution of Ti- $d$ orbitals (green and yellow). They represent the Ti-O bonding states. The Ti- $d$ states are located $2-8 \mathrm{eV}$ above the valence band. From electron counting, there is no electron in Ti-d orbitals. The approximately octahedral crystal-field splitting divides the Ti- $d$ states into nonbonding $t_{2 g}$ states (light green), between $2-5 \mathrm{eV}$ and antibonding $e_{g}$ states (light yellow), between 5-8 eV (see Table I for the common labeling using the $\mathrm{O}_{\mathrm{h}}$ symmetry).

The bottom graph in Fig. 5 shows the sum of the three Ti orbitals, which are adjacent to an oxygen site in the perfect crystal alongside with the total density of states per Ti atom. The Ti orbitals are treated in the local $\mathrm{D}_{3 \mathrm{~h}}$ symmetry of a defect site as introduced in Sec. IIC and Fig. 1. The $A_{1}^{\prime}$ (orange) orbitals are located in the bottom part of the $e_{g}$-like states, while the $E^{\prime}$ (magenta) orbitals contribute to the upper part of the $e_{g}$-like states.

The calculated structural parameters and heats of formation are given in Table II. They are in good agreement with experimental results. The band gap is with $1.905 \mathrm{eV}$ for PBE smaller than the experimental value of $3.05 \mathrm{eV}$ [77]. Conventional functionals such as PBE underestimate the band gap by approximately $1.15 \mathrm{eV}$ [78]. As already noted, this finding is well known for PBE functionals. In the following, the various defects are discussed individually.

\section{Interstitial hydrogen}

The PBE calculations on different charge states on the interstitial $\mathrm{H}$ show that it is only stable in the positive charge state $\mathrm{H}_{\mathrm{i}}^{+}$. Thus, the neutral and negative charge states of the interstitial proton do not exist. The charge state levels for the transition of the neutral and negative charge states lie in the conduction band. This may be due to the band-gap underestimation of PBE.

The interstitial hydrogen $\mathrm{H}_{i}^{+}$forms an $\mathrm{OH}$ bond [as indicated by the arrow in Fig. 6(a)] perpendicular to the Ti-O plane. The $\mathrm{OH}$ bond length is $1.003 \AA$, about $0.1 \%$ smaller than an $\mathrm{OH}$ bond in water at $60 \mathrm{~K}[80]$. The three Ti-O bonds close to this $\mathrm{O}-\mathrm{H}$ bond expand by about $7.2 \%$, resulting in a trigonal pyramidal distortion of the oxygen coordination shown in Fig. 6(a). The $\mathrm{OH}$ group forms a hydrogen bond (marked by dash line) with one of the oxygen ions on the opposite side of the cage. The hydrogen bond of the hydrogen interstitial is $1.773 \AA$ and, therefore, $1.3 \%$ larger than that in water with $1.75 \AA$. The off-axis orientation of the hydrogen bond tilts the $\mathrm{OH}$ bond by $9^{\circ}$ away from the plane normal of the O-Ti bonds. The distance of the defect center $\mathrm{C}_{\mathrm{D}}$ to the three neighboring $\mathrm{Ti}$ atoms reveals outward breathing by about $5.8 \%$.

The density of states for the interstitial hydrogen $\mathrm{H}_{\mathrm{i}}^{+}$is shown in Fig. 6(b) using the same color code as described in Fig. 5. The total density of states of the supercell per Ti atom (grey) is shown alongside with defect symmetry adapted orbitals $A_{1}^{\prime}, E^{\prime}, \delta-e_{g}$, and $\delta-t_{2 g}$.

The $\mathrm{OH} \sigma$-bond state (blue) splits off below the oxygen $2 p$ valence band at about $-6.5 \mathrm{eV}$. The orbital of the $\sigma$ bond is represented in an isosurface plot as depicted in Fig. 6(b). The state extends along with the hydrogen bond to the opposite $\mathrm{O}$ ion. There is no gap state for the interstitial hydrogen. Of particular interest concerning the measured EEL spectra are the defect induced changes of the Ti- $d$ states forming the conduction band. In the low energy part of the $e_{g}$ states at about $5 \mathrm{eV}$, we observe an accumulation of spectral weight. The orbital representation of this state is given as isosurface plot. We attribute the accumulation of $e_{g}$ states at the bottom of the $e_{g}$ band to the electrostatic attraction of the electrons in the Ti levels and the nearby positive defect.

\section{Oxygen vacancy}

In the $\{110\}$ plane of $\mathrm{r}-\mathrm{TiO}_{2}$, each $\mathrm{O}$ atom has an approximately trigonal coordination of three Ti atoms [Fig. 6(c), highlighted with 1,2, and 3]. Thus, the formation of an oxygen vacancy leaves three $\mathrm{Ti}$ ions undercoordinated. The PBE calculations on different charge states show that $\mathrm{O}$ vacancy is only thermodynamically stable in the doubly positive charge state $\mathrm{V}_{\mathrm{O}}^{2+}$. Electrons added to this state are transferred to the conduction band.

For the oxygen vacancy $\mathrm{V}_{\mathrm{O}}^{2+}$, the outward breathing is $12.5 \%$. The breathing seems to follow a simple electrostatic argument: The positive Ti ions repel each other.

The DoS for the supercell with the oxygen vacancy $\mathrm{V}_{\mathrm{O}}^{2+}$ is shown in Fig. 6(d). The DoS graph shows that the doubly positive charge state does not introduce states into the band gap. The Ti- $d$ states with $A_{1}^{\prime}$ character (orange) are shifted with respect to the undoped material, from the $e_{g}$-like continuum downwards to the continuum of the $t_{2 g}$-like states and upwards to the top of the $e_{g}$-like continuum. The corresponding two states with $E^{\prime}$ character (magenta) are nearly degenerate and 


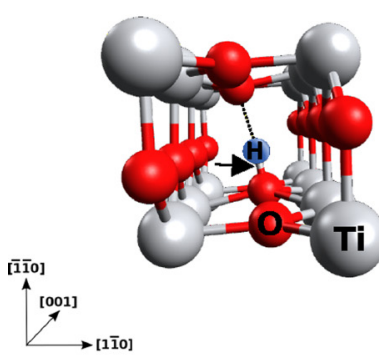

(a)

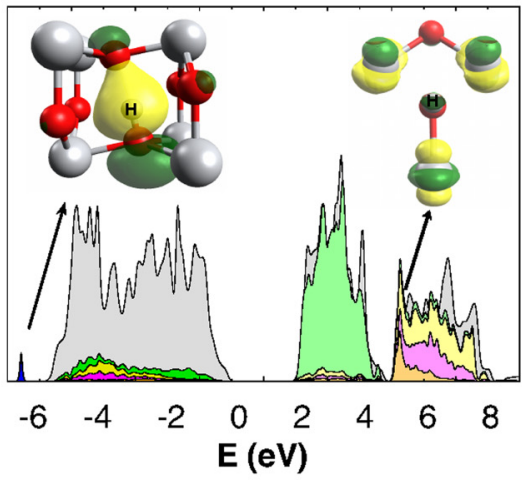

(b)
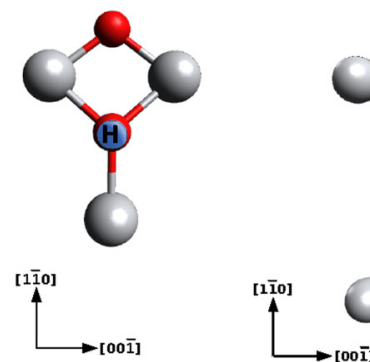

(c)

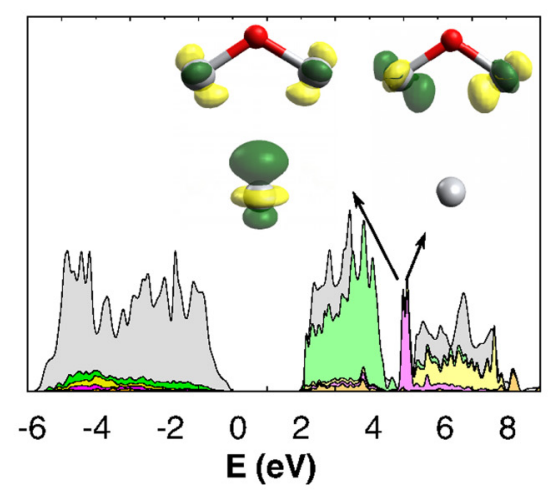

(d)

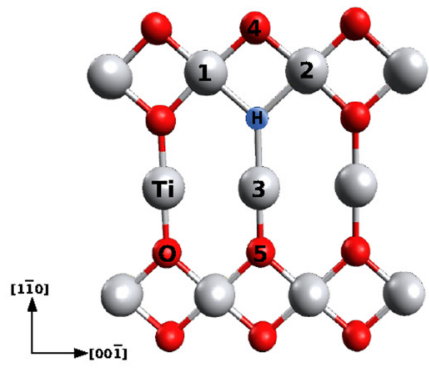

(e)

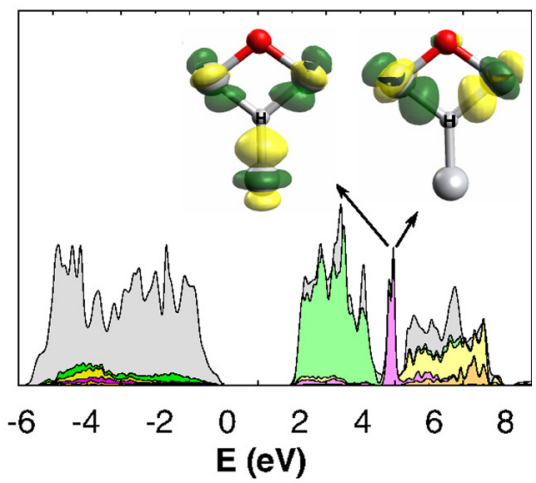

(f)

FIG. 6. Structural and electronic properties of the three stable $\mathrm{H}$-related defects in $\mathrm{r}-\mathrm{TiO}_{2}$. (a) Illustration of the $\mathrm{H}$ position for the positively charged state of the interstitial hydrogen $\left(\mathrm{H}_{\mathrm{i}}^{+}\right)$. Hydrogen bond is represented by dotted line. (b) Density of states of interstitial hydrogen atom $\mathrm{H}_{\mathrm{i}}$ in the positive charge state. The projected density of states is colored as follows: hydrogen (blue), $A_{1}^{\prime}$ (orange), $E^{\prime}$ (magenta), $\delta-e_{g}$ (yellow), and $\delta$ - $t_{2 g}$ (green) orbitals on the three Ti neighbors of the defect. To provide context, the total density of states of $r-\mathrm{TiO}_{2}(\mathrm{grey})$ is shown as well. The projected density of states is related to the colored area, rather than the ordinate value. Relevant wave functions are shown as isosurface with colors (yellow and green) distinguishing the sign. (c) Structure of the oxygen-vacancy ( $\mathrm{V}_{\mathrm{O}}$ ) and (d) density of states of the oxygen vacancy $\mathrm{V}_{\mathrm{O}}$ in the double positive charge state. (e) Structure of the hydrogen-complex $\mathrm{H}_{\mathrm{O}}$ with an oxygen vacancy, in $\mathrm{r}-\mathrm{TiO}_{2}$ and (f) density of states for the hydrogenated oxygen vacancy $\mathrm{H}_{\mathrm{O}}$ in the positive charge state.

form two sharp peaks in the quasi gap between the Ti $t_{2 g}$ and $e_{g}$-like states at $5 \mathrm{eV}$.

In comparison to the undoped $\mathrm{r}-\mathrm{TiO}_{2}$, shown in Fig. 5, a contribution of the $A_{1}^{\prime}$ and the $E^{\prime}$ states, which are derived from $e_{g}$-like states, are now located much lower in energy, namely in the $t_{2 g}$-like continuum. This results from the Ti- $d$ orbitals pointing into the vacancy, which have lost an antibond with the former oxygen neighbor. This antibond was responsible for the crystal-field splitting that shifted these orbitals up, above the $t_{2 g}$-like orbitals in $\mathrm{r}-\mathrm{TiO}_{2}$ (Fig. 5). In the absence of the oxygen ion, the $A_{1}^{\prime}$ and $E^{\prime}$ states are shifted down in energy, so that their energy average lies in the range of the $t_{2 g}$-like bands. The orbital presentations of the two situations of the $E^{\prime}$ states are shown in isosurface plots on top of the DoS in Fig. 6(d).

The $E^{\prime}$ states, in the quasi gap, shift spectral weight of the $e_{g}$ states in the low energy.

\section{Hydrogenated oxygenvacancy}

After describing the isolated defects, we investigate here the pair of these two defects, namely the hydrogenated oxygen vacancy $\mathrm{H}_{\mathrm{O}}$. As shown in Fig. 6(e), the hydrogen atom of the $\mathrm{H}_{\mathrm{O}}$ is located almost at the defect center, respectively the position of the missing oxygen ion.
The PBE calculations on different charge states on the hydrogenated oxygen vacancy show that it is thermodynamically stable only in the positive charge state $\mathrm{H}_{\mathrm{O}}^{+}$.

The corresponding DoS for the hydrogenated oxygen vacancy is shown in Fig. 6(f). The DoS graph resembles that of Fig. 6(d) for the $\mathrm{V}_{\mathrm{O}}^{2+}$. In comparison to the oxygen vacancy, the dominant effect is to shift the defect level of $A_{1}^{\prime}$ character (orange) of the oxygen vacancy downward into the continuum of the valence band. The hydrogenated oxygen vacancy $\mathrm{H}_{\mathrm{O}}^{+}$ also does not have states in the band gap.

The Ti- $d$ states with $E^{\prime}$ symmetry (magenta) exhibit sharp peaks and remain in the quasi gap between the $t_{2 g^{-}}$and the $e_{g}$-like states, but centered in it. With respect to the oxygen vacancy, they are slightly shifted to lower energies. Hence, regarding these states, the hydrogenated oxygen vacancy is very similar to the bare oxygen vacancy. We interpret this again with the loss of the oxygen antibond. With respect to the EELS measurements, these quasi gap states can shift spectra weight. The orbital representation of the $E^{\prime}$ states are shown in the iso-surface plots on top the DoS in Fig. 6(d).

Based on the simulation results, we note that all of the defects deliver a qualitative explanation for a shift of states in the unoccupied part of the DoS, which can explain the EEL measurements. 
TABLE III. Defect states $X_{\sigma}$ of an oxygen site, their energies $E_{\sigma}$ and excess particle numbers (stoichiometric factors) $\eta_{e, \sigma}$ for electrons, $\eta_{H, \sigma}$ for hydrogen atoms, and $\eta_{O, \sigma}$ oxygen atoms. The excess particle numbers are counted relative to an ideal bulk site.

\begin{tabular}{cccccc}
\hline \hline$\sigma$ & $X_{\sigma}$ & $E_{\sigma}-\sum_{k \in\{e, O, H\}} \eta_{k, \sigma} \mu_{k}^{\text {ref }}$ & $\eta_{e, \sigma}$ & $\eta_{H, \sigma}$ & $\eta_{O, \sigma}$ \\
\hline 1 & Bulk & $0.000 \mathrm{eV}$ & 0 & 0 & 0 \\
2 & $\mathrm{H}_{\mathrm{i}}^{+}$ & $-1.59018 \mathrm{eV}$ & -1 & +1 & 0 \\
3 & $\mathrm{~V}_{\mathrm{O}}^{2+}$ & $0.78779 \mathrm{eV}$ & -2 & 0 & -1 \\
4 & $\mathrm{H}_{\mathrm{O}}^{+}$ & $1.90610 \mathrm{eV}$ & -1 & +1 & -1 \\
\hline \hline
\end{tabular}

\section{Defect electrochemistry of the interface and the bulk}

To get a deeper understanding about which of the defects are responsible for the EELS changes, we combine the DFT results with statistical mechanics to simulate the $\mathrm{Pd} / \mathrm{r}-\mathrm{TiO}_{2}$ interface.

The total energies from the DFT calculations allow one to estimate the defect concentrations from a thermodynamic multistate defect (TMD) model. Furthermore, it allows one to link the concentration to the spatial position in the sample. The TMD model is described in Appendix C. It is based on a multistate description for the oxygen-lattice sites. Each state $X_{\sigma}$ corresponds to a specific defect type and charge state. The defects states used in the present study are specified in Table III, along with their energy $E_{\sigma}$ and excess number of electrons, hydrogen and oxygen atoms. The TMD model accounts for the energy and the configurational entropy of the defect distributions. In addition, electrons and holes are considered within the effective-mass approximation [see Eq. (C4)]. The electrostatic interaction between charge carriers and defects is described as mean field obtained from the Poisson equation [see Eq. (C9)].

The purpose of these model calculations is to elucidate three questions, which emerge from the experimental results presented in Sec. III A:

(i) Does the potential step between the interface and the bulk affect the defect concentrations sufficiently to explain the observed spatial profile of the EELS?

(ii) Is there a relevant increase in the defect concentration by pressure increase from 1 to $10 \mathrm{~Pa}$ ?

(iii) Does the interface concentration of defects increase up to a measurable concentration of the order of one per oxygen site in the pressure range of $10 \mathrm{~Pa}$ ?

In the following, we describe the processes, which emerge from our model. The treatment considers the experimental conditions during sample preparation as well as before and after hydrogen exposure.

\section{Bulk oxide before hydrogen exposure}

Figure 7 shows the concentrations of defects and electrons in bulk $\mathrm{r}-\mathrm{TiO}_{2}$ as a function of the oxygen chemical potential $\mu_{\mathrm{O}}$ at room temperature and ambient pressure. Before the $\mathrm{Pd}$ film is deposited, the oxygen chemical potential $\mu_{\mathrm{O}}$ is controlled by the oxygen partial pressure and the temperature of the ambient.

The natural atmospheric hydrogen content of $0.5 \mathrm{ppm}$ is taken into account [81]. Despite the small hydrogen content

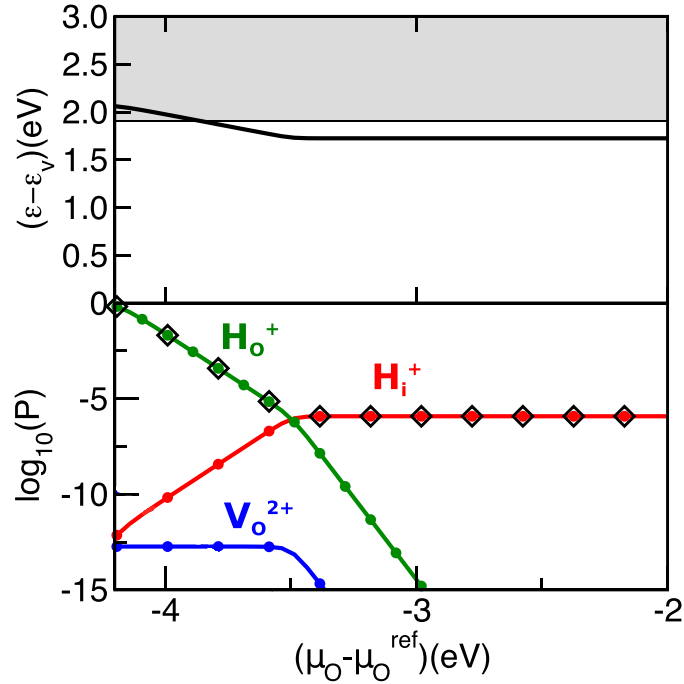

FIG. 7. Calculated Fermi level $\mu_{e}$ relative to the valence band top $\epsilon_{v}$ (top) as well as defect and charge carrier concentrations (bottom) in bulk $\mathrm{r}-\mathrm{TiO}_{2}$ as function of the oxygen chemical potential $\mu_{\mathrm{O}}$. Data are shown for room temperature. The atmospheric hydrogen partial pressure is taken into account. The concentrations in terms of probability $P$ per $\mathrm{O}$ site of interstitial hydrogen $\mathrm{H}_{i}^{+}$are shown in red, those of oxygen vacancies $\mathrm{V}_{\mathrm{O}}^{2+}$ are shown in blue, and those of hydrogenated oxygen vacancies $\mathrm{H}_{\mathrm{O}}^{+}$are shown in green. The concentration of electrons in the conduction band is represented by the open diamonds. The shaded region of the top graph indicates the difference between the experimental and the calculated band gaps. The latter are obtained from the Kohn-Sham levels.

of the atmosphere, its partial pressure is sufficiently high to dominate the defect chemistry of $\mathrm{r}-\mathrm{TiO}_{2}$ at room temperature. Hydrogen related defects dominate for both, oxygen-poor and oxygen-rich conditions.

Figure 7 also summarizes the Fermi level position (top) and charge carrier concentrations (bottom) in bulk $\mathrm{r}-\mathrm{TiO}_{2}$ as a function of the oxygen chemical potential $\mu_{\mathrm{O}}$. The bulk Fermi level is determined by the charge neutrality condition. The Fermi level is measured relative to the valence-band top $\epsilon_{v}$ and the oxygen chemical potential is measured relative to reference $\mu_{\mathrm{O}}^{\mathrm{ref}}:=\frac{1}{2} E\left[\mathrm{O}_{2}\right]$.

Under oxygen-rich conditions, that is above $\mu_{\mathrm{O}}=\mu_{\mathrm{O}}^{\text {ref }}-$ $3.5 \mathrm{eV}$, the dominant defect is the hydrogen interstitial $\mathrm{H}_{\mathrm{i}}^{+}$as shown in Fig. 7. Our model estimates an $\mathrm{H}_{\mathrm{i}}^{+}$concentration (red in Fig. 7) of about $10^{-6}$ per oxygen site or $10^{17} \mathrm{~cm}^{-3}$. The positive charge of the defects is compensated by the conduction electrons. For this oxygen-rich region, the Fermi level lies $0.18 \mathrm{eV}$ below the conduction-band edge.

Under oxygen-poor conditions, that is for effective chemical potentials below $\mu_{\mathrm{O}}=\mu_{\mathrm{O}}^{\text {ref }}-3.5 \mathrm{eV}$, the positive hydrogenated oxygen vacancy $\mathrm{H}_{\mathrm{O}}^{+}$(green in Fig. 7) is the dominant defect. The concentration of hydrogenated oxygen vacancies rises strongly with decreasing oxygen chemical potential. To maintain charge neutrality, also the conduction-electron concentration increases alongside with the defect concentration as marked with open black diamonds. The increasing conduction electron density is reflected by the increasing Fermi level as shown in the top of Fig. 7. 
TABLE IV. Partial pressures and chemical potentials for (1) ambient conditions, (2) high-temperature anneal under oxygen atmosphere, (3) Pd deposition in vacuum, (4) ambient conditions after Pd deposition with frozen vacancy concentration $\mathrm{V}_{\mathrm{O}}$ and $\mathrm{H}_{\mathrm{O}}$, (5) hydrogen exposure $1 \mathrm{~Pa}$, and (6) hydrogen exposure $10 \mathrm{~Pa}$. The chemical potentials are specified as relative values $\Delta \mu_{\mathrm{O}}=\mu_{\mathrm{O}}-\mu_{\mathrm{O}}^{\text {ref }}$ and $\Delta \mu_{\mathrm{H}}=\mu_{\mathrm{H}}-\mu_{\mathrm{H}}^{\mathrm{ref}}$. Numbers 4,5 , and 6 show the equivalent partial pressures, after the $\mathrm{Pd}$ film prohibits oxygen exchange with the atmosphere.

\begin{tabular}{lrcclc}
\hline \hline Nr. & $\mathrm{T}(\mathrm{K})$ & $p_{\mathrm{O}_{2}}(\mathrm{~Pa})$ & $p_{\mathrm{H}_{2}}(\mathrm{~Pa})$ & $\Delta \mu_{\mathrm{O}}(\mathrm{eV})$ & $\Delta \mu_{\mathrm{H}}(\mathrm{eV})$ \\
\hline 1 & 293 & $10^{+5}$ & $5 \times 10^{-2}$ & -0.218 & -0.210 \\
2 & 1173 & $10^{+5}$ & 0 & -1.271 & $-\infty$ \\
3 & 1023 & $7 \times 10^{-3}$ & 0 & -1.806 & $-\infty$ \\
4 & 293 & $0.3 \times 10^{-107}$ & $5 \times 10^{-2}$ & -3.49 & -0.210 \\
5 & 293 & $2 \times 10^{-107}$ & 1 & -3.47 & -0.172 \\
6 & 293 & $6 \times 10^{-107}$ & 10 & -3.45 & -0.143 \\
\hline \hline
\end{tabular}

Below $\mu_{\mathrm{O}}-\mu_{\mathrm{O}}^{\mathrm{ref}}=-4.2 \mathrm{eV}$, our model predicts defect concentrations of the order of one per oxygen site (green curve in Fig. 7), which marks the limit of its applicability. Such large defect concentrations indicate that the material may be close to a phase transition.

The effective oxygen chemical potential in the material is strongly preparation dependent because the oxygen exchange with the atmosphere is effectively suppressed at room temperature. The exchange requires firstly sufficiently large defect mobility, and secondly an effective surface reaction such as the oxidation of oxygen vacancies at the surface. Both are not present at room temperature [82-84]. Therefore, the material memorizes the defect concentration of the last high-temperature preparation step, which allowed the oxide to exchange oxygen effectively with the surrounding atmosphere.

In the following, we estimate the defect concentrations originating from the experimental treatments. In our experiment, the $\mathrm{r}-\mathrm{TiO}_{2}$ crystals were first annealed at $1173 \mathrm{~K}$ at approximately $10^{5} \mathrm{~Pa} \mathrm{O}_{2}$. Subsequently, they have been cooled in the same oxygen atmosphere. Then, they were kept under ambient conditions for several hours or days before the Pd films were deposited at $1023 \mathrm{~K}$ and at $\mathrm{O}_{2}$ partial pressure of $7 \times 10^{-3} \mathrm{~Pa}$. The samples remained in this environment for half an hour before they were cooled down in the same atmosphere. The material is then stored under ambient conditions. After preparation, the TEM lamella was transferred to the ETEM, where it has been kept for about $12 \mathrm{~h}$ in vacuum conditions of $10^{-4} \mathrm{~Pa}$. The sample was then exposed to a fixed hydrogen partial pressure of 1 or $10 \mathrm{~Pa}$ of $\mathrm{H}_{2}$. After one hour of hydrogen exposure, the EELS measurement was carried out.

In Table IV, the experimental conditions are translated into oxygen chemical potentials as described in Appendix D. We assume that thermodynamic equilibrium can be reached under the chosen annealing conditions, while the Pd film prevents oxygen exchange with the atmosphere.

As a reference, we list the partial pressures and chemical potentials under ambient conditions. The hydrogen chemical potential was determined by using the natural content of 0.5 ppm of hydrogen in the air.
The oxygen content of the oxide, i.e., its oxygen vacancy concentration, is determined in the high-temperature processing steps. Our thermodynamic model predicts via Eq. (C8) a concentration of $2 \times 10^{-7}$ oxygen vacancies per site for the $1173 \mathrm{~K}$ annealing at $10^{5} \mathrm{~Pa}$ and of $7 \times 10^{-7}$ vacancies per site for the Pd deposition at $1023 \mathrm{~K}$ in a vacuum.

We assume that the oxygen-vacancy concentration is effectively maintained after the Pd deposition at high temperatures, because the Pd film prevents diffusion of oxygen from the atmosphere into the sample. Therefore, we assume a remaining oxygen vacancy concentration, hydrogenated or not, of $7 \times 10^{-7}$ per oxygen site after sample preparation.

After deposition of the Pd film, the total concentration of oxygen vacancies, hydrogenated and not, is frozen in. Therefore, while the material cools down to room temperature, the oxygen chemical potential moves downward. At room temperature, the concentration of $7 \times 10^{-7}$ oxygen vacancies, present during Pd deposition, translates into an oxygen chemical potential of $\mu_{\mathrm{O}}=\mu_{\mathrm{O}}^{\mathrm{ref}}-3.5 \mathrm{eV}$. For a hypothetical environment that can exchange oxygen with the probe, the oxygen chemical potential, respectively the oxygen vacancy concentration, translates into an ultra-low equivalent partial pressure of $10^{-107} \mathrm{~Pa}$.

As seen in Fig. 7, after the sample preparation the material is thus at the boundary $\mu_{\mathrm{O}}=\mu_{\mathrm{O}}^{\text {ref }}-3.5 \mathrm{eV}$ between electron-rich conditions, dominated by interstitial hydrogen, and electron-poor conditions, dominated by hydrogenated oxygen vacancies.

The Pd film is further important as a catalyst for hydrogen dissociation [43]. As the thin TEM lamella is exposed to air, hydrogen can diffuse into it and saturate the existing oxygen vacancies, which results in the defect concentrations presented in Fig. 7.

\section{Defect profile at the metal-oxide interface}

Above we considered the defect concentrations in the bulk, where they are governed by local charge neutrality. In the following, we discuss the interface and the influence on hydrogen loading.

While the analysis predicts an equal concentration of hydrogenated oxygen vacancies $\mathrm{H}_{\mathrm{O}}^{+}$and interstitial hydrogen $\mathrm{H}_{\mathrm{i}}^{+}$, interstitial hydrogen is the dominant defect under hydrogen exposure. This allows us to simplify the following discussion by limiting it to a single defect, namely $\mathrm{H}_{\mathrm{i}}^{+}$, albeit the calculations have been performed with the complete model.

With the above-mentioned limitation, the $\mathrm{H}_{\mathrm{i}}^{+}$defect concentration per oxygen site, $P_{\mathrm{H}^{+}}$, can be expressed in terms of the hydrogen chemical potential $\mu_{\mathrm{H}}$ and the Fermi level $\mu_{e}$ [Eq. (C8)],

$$
P_{\mathrm{H}_{\mathrm{i}}^{+}}=\left[1+e^{\beta\left(E\left[\mathrm{H}_{\mathrm{i}}^{+}\right]-E[B]+\left(\mu_{e}-v_{e}(\vec{r})\right)-\mu_{\mathrm{H}}\right)}\right]^{-1},
$$

where $v_{e}(\vec{r})=-e \Phi(\vec{r})$ is the potential acting on the electrons due to the electric potential $\Phi(\vec{r})$. Per convention, $v_{e}(\vec{r})$ is equal to the energy of the local valence-band top.

The energy $\mu_{e}-v_{e}(\vec{r})$ is required to lift the electron to the Fermi level, when the charged defect $\mathrm{H}_{\mathrm{i}}^{+}$is formed from the neutral $\mathrm{H}_{\mathrm{i}}^{(0)}$. This energy differs between the bulk and the interface and, therefore, also the concentrations are different. 
Increasing the hydrogen partial pressure of the environment enhances the bulk concentration of $\mathrm{H}_{i}^{+}$. The charge neutrality condition pushes the Fermi level upward, which in turn increases the number of electrons in the conduction band. The high position of the Fermi level counteracts the formation of additional charged defects until charge neutrality is reached.

At the interface, this mechanism works entirely different. Rather than lifting the electron towards a Fermi level near the conduction-band edge, it can be placed into the metal. At the interface, the Fermi level is pinned by the Schottky barrier at a specific energy $\Phi_{B n}$ below to the local conduction band of the oxide. This energy $\mu_{e}-v_{e}\left(\vec{r}_{I}\right)=\epsilon_{c}-\Phi_{B n}$ lies further below the local conduction-band edge $\epsilon_{c}+v_{e}(\vec{r})$ than in the bulk, which stabilises positively charged defects near the interface. For sufficiently low defect concentrations, the enhancement of defects at the interface can be related to the band bending $\Delta v_{e}=v_{e}(z=0)-v_{e}(z=\infty)$, where $z$ is the distance from the interface, as $e^{\beta \Delta v_{e}}$. Approximating the band bending by the Schottky barrier yields an interface enhancement of the defect concentration by a factor $10^{13}$ relative to that of the bulk oxide.

\section{Hydrogen loading}

Hydrogen related changes of the EEL spectrum are observed in the experiment for a concentration of hydrogen related defects per oxygen site in the order of $10 \%$, as estimated from the shift of $0.15 \mathrm{eV}$ in the measured EELS [Fig. 4(b)] and the defect induced shift of calculated energy levels in the range 1-2 eV (Fig. 6).

The crossover to a hydrogen concentration of order 1 is estimated from Eq. (3) as the hydrogen chemical potential $\mu_{\mathrm{H}}=$ $E\left[H_{i}^{+}\right]-E[B]+\mu_{e}-v_{e}(\vec{r})$. At the interface this value is reached for $\mu_{H}^{*}=\mu_{H}^{\text {ref }}-0.47 \mathrm{eV}$, whereas in the bulk it is reached substantially later, namely at $\mu_{H}^{*}=\mu_{H}^{\mathrm{ref}}+0.31 \mathrm{eV}$. This reflects the large difference of hydrogen related defects at the interface and in the bulk. Experimentally, this crossover takes place above a hydrogen chemical potential of $\mu_{\mathrm{H}}=$ $\mu_{\mathrm{H}}^{\mathrm{ref}}-0.143 \mathrm{eV}$, corresponding to a hydrogen partial pressure of $10 \mathrm{~Pa}$.

There is a discrepancy between the experimentally observed crossover at $\mu_{H}^{*}>\mu_{H}^{\text {ref }}-0.143 \mathrm{eV}$ (see Table IV) and the prediction from the TMD model of $\mu_{H}^{*}=\mu_{H}^{\text {ref }}-0.47 \mathrm{eV}$. We attribute this discrepancy of $0.33 \mathrm{eV}$ to the uncertainties of the parameters used in the TMD model. The most likely source of error is the underestimation of the band gap by $1.1 \mathrm{eV}$ in DFT. This underestimates the energy $E_{\sigma}$ of positive defects. Furthermore, the band-gap underestimation hides defect-induced charge state levels below the conduction-band edge. These charge state levels may pin the Fermi level and thus limit the concentration of positively charged defects. This in turn, would reduce the band bending and consequently the interface enhancement of positively charged defects. A second possible source of errors is the value of the Schottky barrier, which has not been directly measured but deduced from the Schottky model. In view of these uncertainties, we attribute the deviation of $\mu_{H}^{*}$ between theory and experiment to the limitations of the theory.

Based on our findings, the inhomogeneous defect distribution in $z$ is the effect of positively charged mobile hydrogen

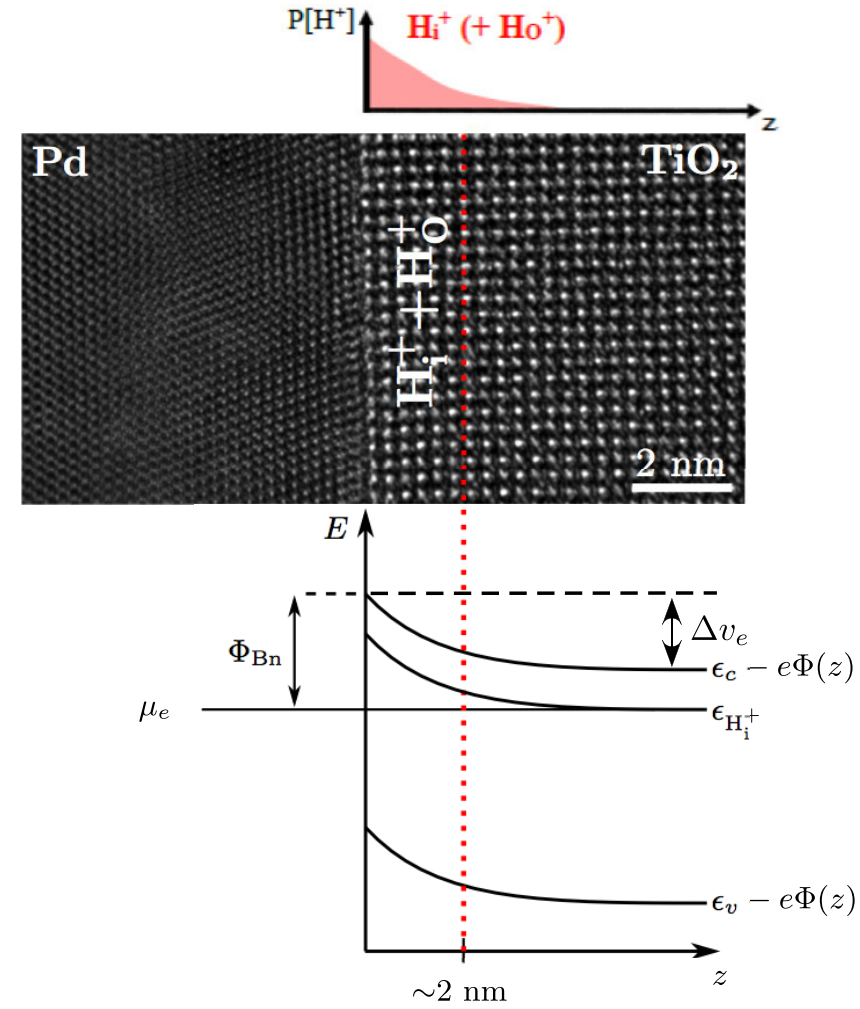

FIG. 8. Schematic diagram of the electronic structure of the $\mathrm{Pd}(111) / \mathrm{TiO}_{2}(110)$ close to the interface in hydrogen environment. Due to the presence of the Schottky barrier $\Phi_{\mathrm{Bn}}$, hydrogen-related defects enrich at the interface and its vicinity. Mainly the concentration of interstitial hydrogen $\mathrm{H}_{\mathrm{i}}$ is influenced by the applied hydrogen partial pressure within the ETEM.

species. From these findings, we derived a schematic band picture as shown in Fig. 8. The corresponding hydrogenrelated defects are present in the $2 \mathrm{~nm}$ vicinity of the $\mathrm{Pd} / \mathrm{TiO}_{2}$ interface.

\section{SUMMARY AND CONCLUSIONS}

In a combined study using ELNES, ETEM, and DFT we investigated the $\mathrm{Pd} / \mathrm{r}-\mathrm{TiO}_{2}$ interface under various hydrogen partial pressures. The energy splitting between $b^{\prime}$ and $a$ bands in the Ti $L_{3}$ ELNES was found to be decreased for hydrogen partial pressures exceeding $10 \mathrm{~Pa}$. This effect is limited to $2 \mathrm{~nm}$ from the interface.

The calculated DoS of defects $\left(\mathrm{H}_{\mathrm{i}}^{+}, \mathrm{V}_{\mathrm{O}}^{2+}\right.$, and $\left.\mathrm{H}_{\mathrm{O}}^{+}\right)$qualitatively explain the origin of these hydrogen-induced changes of the ELNES. The shift or the antibonding Ti- $d$ orbitals is attributed to the Coulomb potential from the positive defect located at an adjacent oxygen site.

The defect distribution has been studied with a TMD model using energies from DFT calculations. The TMD model predicts the spatial distribution of defects in thermal equilibrium and the resulting electrostatic potential near a metal-semiconductor interface. Thus, it generalizes the classical metal-semiconductor theory, which studies stationary dopants [58].

The TMD model predicts that, despite the small hydrogen concentration below the ppm range in the atmosphere, 
hydrogen-related defects $\left(\mathrm{H}_{\mathrm{i}}^{+}\right.$and $\left.\mathrm{H}_{\mathrm{O}}^{+}\right)$are the dominant defects in bulk $\mathrm{TiO}_{2}$. We expect this to be valid also for a broader class of materials. The defect $\mathrm{H}_{\mathrm{O}}^{+}$dominates in oxygen-poor conditions, while the interstitial hydrogen $\mathrm{H}_{\mathrm{i}}^{+}$is the dominant defect under oxygen rich conditions.

An analysis of the processing steps with the TMD model estimates that our samples are at the transition from the oxygen poor regime, where the hydrogenated oxygen vacancy (substitutional hydrogen) $\mathrm{H}_{\mathrm{O}}^{+}$dominates, and the oxygen-rich regime, where interstitial hydrogen $\mathrm{H}_{\mathrm{i}}^{+}$is the dominant defect. This implies that interstitial hydrogen $\mathrm{H}_{\mathrm{i}}^{+}$becomes the dominant defect, when the hydrogen partial pressure is increased.

According to the TMD model, the concentration of positively charged defects is enhanced at the interface compared to the bulk oxide. This increases, in particular, the interface concentration of the dominant hydrogen related defect, namely $\mathrm{H}_{\mathrm{i}}^{+}$.

Increasing the hydrogen partial pressure to $10 \mathrm{~Pa}$, as in our EELS experiments within the ETEM, increases the interstitial hydrogen defect concentration further. Changes in the EELS are expected to become visible at an interfacial defect concentration in the range of $10 \%$. Such a defect concentration is compatible with our calculations, even though the required hydrogen chemical potential is predicted $0.33 \mathrm{eV}$ lower than observed in experiment.

The defect density in $\mathrm{r}-\mathrm{TiO}_{2}$ is a decaying distribution of positively charged mobile hydrogen species starting from interface to the bulk. The presence of the defect states can explain an increased conductivity of the metal/TiO ${ }_{2}$ contacts in hydrogen gas [20-22]. Strungaru et al. [22] explain gas sensing measurements on platinum $/ \mathrm{TiO}_{2}$ contacts with the presence of interstitial hydrogen and hydrogen-oxygen vacancy complexes. Based on the presented results we suggest that interstitial hydrogen is responsible for the changed electrical properties of the metal/semiconductor contact.

The present paper offers an example of hydrogen interacting with the $\mathrm{Pd} / \mathrm{r}-\mathrm{TiO}_{2}$ model system. Hydrogen induced changes in the close vicinity of the $\mathrm{Pd} / \mathrm{r}-\mathrm{TiO}_{2}$ interface, give important insights for understanding gas sensing and catalytic processes of buried interfaces. The described change of the mobile defect concentration at metal/oxide interfaces also concerns the perimeter of catalytically active metal particles supported by an oxide or vice versa. With this paper, we open a door to a view on the physics at interfaces, that include the behavior of mobile defect species.

\section{ACKNOWLEDGMENTS}

We thank fruitful discussions with Thorsten Stolper and Felix Jung. We acknowledge the help of Niklas Herwig for AFM and XPS, as well as Julius Scholz for XPS measurements. The use of equipment in the Collaborative Laboratory and User Facility for Electron Microscopy (CLUE) [85] is gratefully acknowledged. Financial supports from the CRC 1073 (Projects B03, C03, C04, C06, and Z02), PU131/9-2, and Project ID 390874152 (POLiS Cluster of Excellence) of the Deutsche Forschungsgemeinschaft (DFG) are gratefully acknowledged. J.C. acknowledges financial support from the Czech Science Foundation (Project 21-16218S).

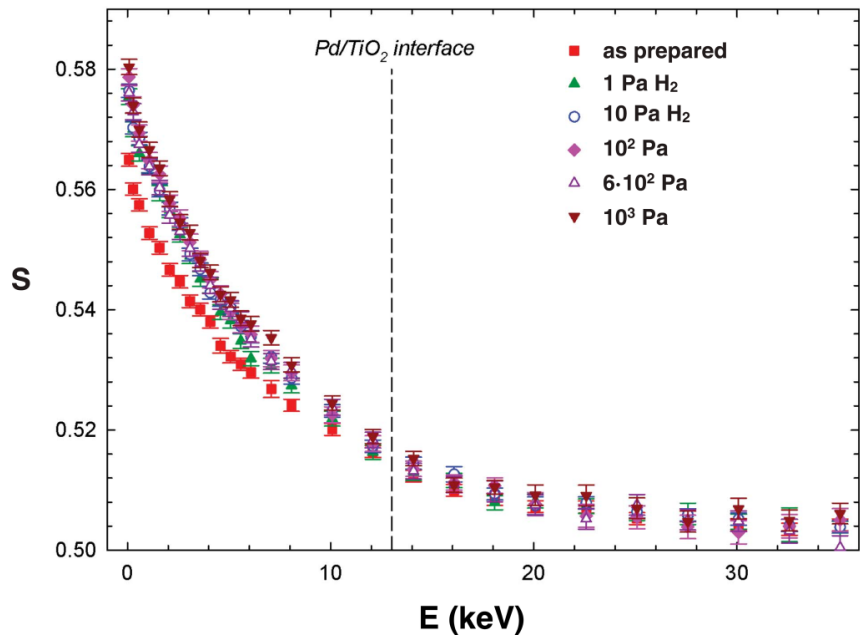

FIG. 9. VEPAS on the $\mathrm{Pd} / \mathrm{r}-\mathrm{TiO}_{2}$ system shows the $S$ parameter as a function of the positions incident energy $E$. No changes of the $S$ parameter are found at the interface or in the $\mathrm{r}-\mathrm{TiO}_{2}$ bulk.

\section{APPENDIX A: VARIABLE ENERGY POSITRON ANNIHILATION SPECTROSCOPY (VEPAS)}

Variable energy positron annihilation spectroscopy (VEPAS) [86] was employed to complementary characterize the defect depth profile in hydrogen gas exposed $\mathrm{Pd} / \mathrm{r}-\mathrm{TiO}_{2}$ samples. The results are shown in Fig. 9. The VEPAS studies were carried out on a magnetically guided slow positron beam [87]. The energy of incident positrons was varied in the range from $80 \mathrm{eV}$ up to $35 \mathrm{keV}$. The doppler broadening of the annihilation photopeak was measured by a HPGe detector with the energy resolution of $1.09 \mathrm{keV}$ at $511 \mathrm{keV}$. The shape of the Doppler broadened annihilation photopeak was characterized using the $S$ (sharpness) parameter [63]. At very low incident energies $(E<1 \mathrm{keV})$ positrons annihilate almost exclusively on the sample surface. With increasing energy, positrons penetrate deeper and deeper into the $\mathrm{Pd}$ over-layer and the fraction of positrons diffusing back to the surface decreases. A further increase of the incident energy $(E>13 \mathrm{keV})$ allows positrons to penetrate into the $\mathrm{r}-\mathrm{TiO}_{2}$ region. Finally, at high incident energies $(E>30 \mathrm{keV})$ virtually all positrons are annihilated in $\mathrm{r}-\mathrm{TiO}_{2}$ and $S$ reaches a bulk value. No changes of the bulk $S$ value caused by hydrogen loading (up to $\mathrm{H}_{2}$ pressure of $10 \mathrm{mbar}$ ) were observed at high energies. Similarly, no changes of $S$ were found at the $\mathrm{Pd} / \mathrm{TiO}_{2}$ interface at a positron energy of $E \approx 13 \mathrm{keV}$ corresponding to the mean penetration depth of $200 \mathrm{~nm}$. This testifies that hydrogen loading did not introduce any defects capable of positron trapping neither into the $\mathrm{r}-\mathrm{TiO}_{2}$ bulk nor into the $\mathrm{Pd} / \mathrm{r}-\mathrm{TiO}_{2}$ interface. Note that only electrically neutral or negatively charged defects are capable of positron trapping while positively charged defects repel positrons and cannot be observed by VEPAS. In addition, enough open volume is needed for positron trapping. Oxygen vacancies are too small to trap positrons, whereas Ti vacancies yield enough open volume. This was investigated by positron density simulations in $\mathrm{r}-\mathrm{TiO}_{2}$ [61]. Hence, from the VEPAS results one can conclude that hydrogen loading did not introduce $\mathrm{Ti}$ vacancies but no conclusion about 


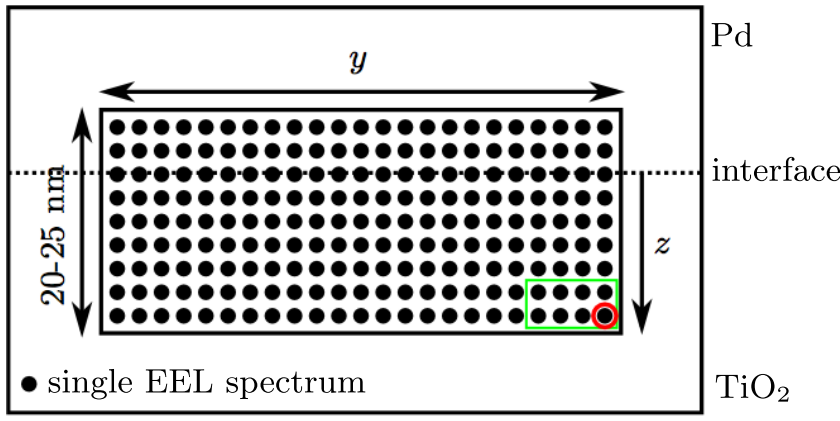

FIG. 10. Scheme of the rectangular SI pattern in the $\mathrm{Pd} / \mathrm{TiO}_{2}$ interface region. The dots represent the positions where EEL spectra are recorded (beam diameter $\approx 5 \AA$ ). Their number depends on the chosen size of the SI pattern and the used step size. $z$ defines the distance to the interface. $y$ varies between $20 \mathrm{~nm}$ and $40 \mathrm{~nm}$. The red circle indicates the start position of the measurement. It first moves in negative $z$ direction and than continues in the same way in the next row to the left. The green box indicates the spectra that are binned afterwards to improve the signal-to-noise ratio.

oxygen vacancies can be made. However, changes of the $S$ parameter are found in the Pd over-layer $(\mathrm{E}<13 \mathrm{keV})$. This effect is discussed by Roddatis et al. [16].

\section{APPENDIX B: ADVANCED EELS PROCEDURE}

For the EELS studies at the interface, a procedure was necessary that combines high energy resolution, low energy drift and a good signal to noise ratio. In contrast to other suggested methods $[88,89]$, the procedure presented here offers error bars on electron energy-loss (EEL) spectra. Rectangular spectrum imaging (SI) pattern are chosen to measure a whole set of EEL spectra on the flat and sharp interfaces. This pattern is indicated schematically in Fig. 10. The measurement protocol describes as follows: A SI pattern is placed on the region of interest, which is, here, at the $\mathrm{Pd} / \mathrm{TiO}_{2}$ interface, see Fig. 10. Thus, the corresponding EEL spectra are collected at defined positions, which are marked with red dots in Fig. 10. The electron beam starts scanning at the red marked position and continues in negative $z$ direction. The $z$ coordinate is defined as the distance to the $\mathrm{Pd} / \mathrm{TiO}_{2}$ interface. This interface $(z=0)$ is here chosen as that location where the EEL spectrum does not contain any significant Ti $L$ edge signal. Afterwards, the measurement continues in the same way in the next row to the left. Vertical alignment of highest interface sharpness was provided by studying a tilting series. The lateral interface homogeneity was verified by repeated measurements at different interface positions. Assuming lateral interface homogeneity, the important changes are here expected just to depend on $z$. This allows binning the acquired EEL spectra (as indicated with the green box) to enhance the signal-to-noise ratio $(\mathrm{S} / \mathrm{N})$.

Details on the measurements and data evaluation is described in the following. A more detailed description is given by Bongers [61].

(1) The Ti $L$ edge is studied by EELS in monochromated scanning TEM (STEM) mode (energy resolution $200 \mathrm{meV}$, probe size $\approx 5 \AA$ ) as a function of the distance to the interface $z$ and of the $\mathrm{H}_{2}$ pressure. The final energy resolution of the EEL spectra in the $\mathrm{r}-\mathrm{TiO}_{2}$ was better than $500 \mathrm{meV}$. Beam currents and integration times are about $130 \mathrm{pA}$ and about $50 \mathrm{~ms}$ per EEL spectrum. The choice of a relatively low integration time (seconds can easily be achieved) for the core loss region results in low energy drift of the total spectrum and allows for a good energy alignment with Dual EELS. The low-loss region is used for this alignment. The chosen dispersions for Dual EELS are $0.05 \mathrm{eV} / \mathrm{ch}$ and $0.1 \mathrm{eV} / \mathrm{ch}$. A rectangular SI pattern (compare Fig. 10), aligned perpendicular to the interface, is used to record the EEL spectra with step sizes of $0.5 \mathrm{~nm}$ and $1 \mathrm{~nm}$. The total collection time for one SI pattern is about $60 \mathrm{~s}$ to minimize spatial sample drift effects. A spatial sample drift correction is, furthermore, performed after every fourth row.

(2) To get an appropriate $\mathrm{S} / \mathrm{N}$ ratio, the data are preferentially binned parallel to the interface. This is indicated with the green box in Fig. 10. The binning is done by a script introduced in the Gatan Microscopy Suite DigitalMicrograph (v2.32.888.0, 32-bit). The binned EEL spectra are processed further in Digital Micrograph according to the following steps: Zero-loss centering using the reflected tail method, pre-edge background subtraction via an inverse power-law function with a fitting window from $407 \mathrm{eV}$ to $452 \mathrm{eV}$ and subsequent deconvolution of plasmon contribution by the Fourier-ratio method [90].

(3) A self developed MATLAB script is used for the further data evaluation. A Hartree-Slater cross section (exported from DigitalMicrograph for the Ti $L$ edge) is subtracted from the Ti $L_{3,2}$ edge to compensate for the $L_{3}-L_{2}$ overlap. Figure 2(b) shows the resulting Ti $L_{3,2}$ electron energy-loss near-edge structure (ELNES). Five Lorentz functions are fitted to the five $\mathrm{Ti} L_{3,2}$ ELNES peaks and the resulting envelope function is used to evaluate the physical parameters. An example of an evaluated parameter is the energy splittings $\mathrm{d} E\left(b^{\prime}-a\right)$. The parameters for the same $z$ position are finally evaluated to calculate the mean value and the standard deviation.

(4) Alternatively, all EEL spectra with the same $z$ distance to the interface can be binned and averaged to one EEL spectrum. This "more classical" approach allows to easily calculate the difference spectra shown in Fig. 4 by the sacrifice of the error bar.

In vertical direction the surface of the TEM-lamella was covered with $\mathrm{Pd}$, allowing for dissociative chemisorption of the hydrogen gas molecules. In lateral direction the lamella offers two uncovered $\mathrm{TiO}_{2}$ surfaces. Hydrogen entry from these sides is not expected.

It should be noted that the substrate was monitored by atomic force microscope (AFM) after pretreatment and before Pd deposition (see Fig. 11), yielding clear flat terraces related to the substrate miscut. It shows about 50 atomic steps over $5 \mu \mathrm{m}$ substrate length. This leads to a flat terrace width of $100 \mathrm{~nm}$. The TEM lamella is of much smaller size and should not contain more than 2 terraces, meaning one atomic step.

X-ray photoemission (XPS) also was performed to verify the clean substrate after substrate pretreatment. The oxygen $1 s$ peak is shown in Fig. 12. After pretreatment, it shows the expected spectrum for a clean surface. The initial surface contains contributions coming from $\mathrm{C}-\mathrm{O}, \mathrm{C}-\mathrm{H}$, or $\mathrm{H}-\mathrm{O}-\mathrm{H}$ surface species. 


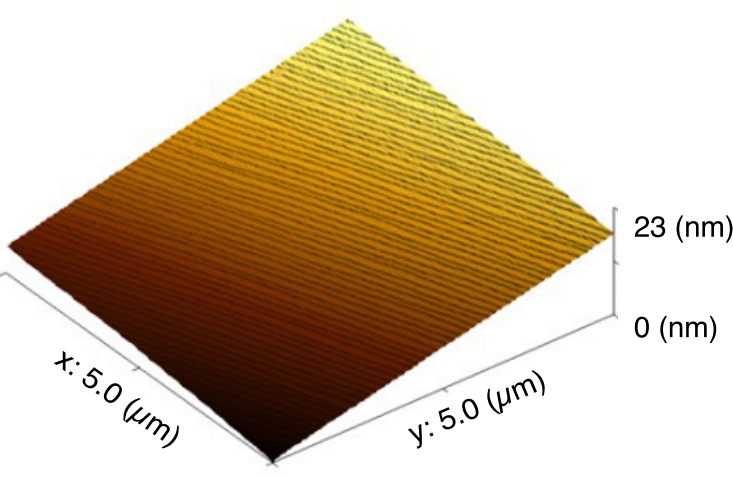

FIG. 11. $25 \mu \mathrm{m}^{2}$ 3D AFM height profile of the $\mathrm{r}-\mathrm{TiO}_{2}$ crystal surface, after pretreatment. The surface shows flat terraces of about $100 \mathrm{~nm}$ width with atomic steps in between.

\section{APPENDIX C: TMD MODEL FOR DEFECT CONCENTRATIONS}

Defect concentrations (probabilities) can be calculated from the defect energies including the configurational entropy of the defect distribution via the grand potentials of the bulk $\left(\Omega_{1}\right)$ and at the interface $\left(\Omega_{1}\right.$ and $\left.\Omega_{2}\right)$. The electrostatic interaction is treated on a mean-field level by electrostatic potential of the defect charge densities. The details are described in the following.

We consider a multistate model, where an oxygen site can be in one of four states $X_{\sigma}$ (see Table III). These states can either be an oxygen atom or one of the defects in its respective charge state at an oxygen site. The interstitial hydrogen is also attributed to an oxygen site.

The grand potential $\Omega$ is a sum over all microstates, each of which is described by a vector $\vec{\sigma}$. Each component $\sigma_{j}$ of this vector selects the state of the particular oxygen site at position $\vec{R}_{j}$ in the $\mathrm{r}-\mathrm{TiO}_{2}$ lattice. The integer value $\sigma_{j}$ selects one of the possible states $X_{\sigma}$ of an oxygen site. The grand potential of

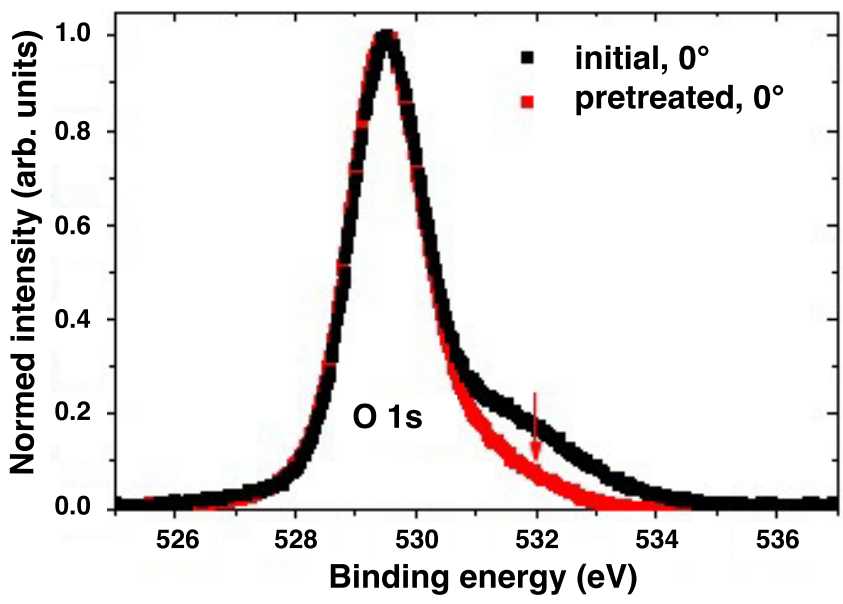

FIG. 12. X-ray photoelectron spectra of the $\mathrm{r}-\mathrm{TiO}_{2}$ substrate surface at an incident angle of $0^{\circ}$. The oxygen $1 s$ edge is shown before and after the pretreatment. The oxygen 1s edge has a shoulder, which decreased after the pretreatment. the defects is given by

$$
\Omega_{1}=-k_{\mathrm{B}} T \ln \sum_{\vec{\sigma}} \exp \left[-\beta\left(\mathcal{E}_{\vec{\sigma}}-\sum_{k \in\{e, \mathrm{O}, \mathrm{H}\}} \mu_{k} \mathcal{N}_{k, \vec{\sigma}}\right)\right],
$$

where $\beta=1 /\left(k_{\mathrm{B}} T\right)$. The system is coupled to particle reservoirs with chemical potentials $\mu_{e}$ for electrons, $\mu_{\mathrm{H}}$ for hydrogen, and $\mu_{\mathrm{O}}$ for oxygen.

The total energies $\mathcal{E}_{\vec{\sigma}}$ and particle numbers $\mathcal{N}_{\vec{\sigma}}$ of the defect distributions are

$$
\begin{aligned}
\mathcal{E}_{\vec{\sigma}} & =\sum_{j}\left(E_{\sigma_{j}}+\eta_{e, \sigma_{j}} v_{e}\left(R_{j}\right)\right), \\
\mathcal{N}_{k, \vec{\sigma}} & =\sum_{j} \eta_{k, \sigma_{j}},
\end{aligned}
$$

where $\eta_{k, \sigma}$ with $k \in\{e, \mathrm{O}, \mathrm{H}\}$ are particle numbers for the specified defect as listed in Table III. The particles considered are electrons, hydrogen atoms and oxygen atoms.

$v_{e}(\vec{r})=-e \Phi(\vec{r})$ is the potential acting on the electrons due to the electric potential $\Phi(\vec{r})$. It is treated within the mean field theory: it plays the role of an external potential, which is later connected self-consistently to the charge density.

In addition, we consider the charge carriers like electrons and holes, which are not related to defects. We start with the grand potential of noninteracting electrons with a density of states $D(\epsilon)$.

$$
\begin{aligned}
\Omega_{2}= & -k_{\mathrm{B}} T \int_{-\infty}^{\infty} d \epsilon \int d^{3} r \frac{1}{V} D\left(\epsilon-v_{e}(\vec{r})\right) \\
& \times \ln \left[1+\exp \left(-\frac{\left|\epsilon-\mu_{e}\right|}{k_{\mathrm{B}} T}\right)\right] .
\end{aligned}
$$

The energy for the filled Fermi sea has been subtracted, which is accounted for by taking the absolute value of $\epsilon-\mu_{e}$. The expression is limited to Fermi levels within the band gap of the oxide.

The functional form of the density of states $D(\epsilon)=$ $D_{v}(\epsilon)+D_{c}(\epsilon)$ is defined, like conduction-band edge $\epsilon_{c}$ and the valence-band edge $\epsilon_{v}$, in the absence of an electric potential. The latter is accounted for by the choice of the argument. The density of states is approximated by parabolic bands for valence and conduction bands with multiplicities $N_{v}$ and $N_{c}$ and effective masses $m_{v}^{*}$ and $m_{c}^{*}$ :

$$
\begin{array}{ll}
D_{c}(\epsilon)=2 \pi V N_{c}\left(\frac{\sqrt{2 m_{c}^{*}}}{2 \pi \hbar}\right)^{3} \sqrt{\epsilon-\epsilon_{c}} & \text { for } \epsilon>\epsilon_{c}, \\
D_{v}(\epsilon)=2 \pi V N_{v}\left(\frac{\sqrt{2 m_{v}^{*}}}{2 \pi \hbar}\right)^{3} \sqrt{\epsilon_{v}-\epsilon} & \text { for } \epsilon<\epsilon_{v} .
\end{array}
$$

The volume $V$ in this expression is canceled in Eq. (C3). The effective masses have been set to $m_{c}^{*}=m_{e}$ for the conduction-band minimum and $m_{v}^{*}=3.33 m_{e}$ for the valenceband maximum [91]. A one to two average of the effective masses calculated with GGA [66] for the $\Gamma \rightarrow Z$ and $\Gamma \rightarrow$ $M / Z$ directions has been used. The multiplicities from our calculated band structure are $N_{c}=4$ for the conduction-band minimum and $N_{v}=2$ for the valence-band top, including spin multiplicity. 
When evaluating the grand potential of free carriers, we retain only the leading term in the Boltzmann factor $\exp \left(-\beta\left|\epsilon-\mu_{e}\right|\right)$.

After adding the grand potentials of the defects and free charge carriers, we obtain the grand potential $\Omega=\Omega_{1}+\Omega_{2}$ for a given potential $v_{e}(\vec{r})$ as

$$
\begin{aligned}
\Omega(T, & \left.\mu_{e}, \mu_{\mathrm{H}}, \mu_{\mathrm{O}},\left[v_{e}\right]\right) \\
= & \int d^{3} r\left\{-\frac{k_{\mathrm{B}} T}{\mathrm{~V}_{\mathrm{O}}}\right. \\
& \times \ln \left(\sum_{\sigma=1}^{N_{s p}} \exp \left[-\beta\left(E_{\sigma}+\eta_{e, \sigma} v_{e}(\vec{r})-\sum_{k \in\{e, \mathrm{O}, \mathrm{H}\}} \eta_{k, \sigma} \mu_{k}\right)\right]\right) \\
& -\left(\frac{m_{e}}{2 \pi \hbar^{2}}\right)^{\frac{3}{2}}\left(k_{\mathrm{B}} T\right)^{\frac{5}{2}}\left[N_{c}\left(\frac{m_{c}^{*}}{m_{e}}\right)^{\frac{3}{2}} e^{-\beta\left(\epsilon_{c}+v_{e}(\vec{r})-\mu_{e}\right)}\right. \\
& \left.\left.+N_{v}\left(\frac{m_{v}^{*}}{m_{e}}\right)^{\frac{3}{2}} e^{-\beta\left(\mu_{e}-\epsilon_{v}-v_{e}(\vec{r})\right)}\right]\right\} .
\end{aligned}
$$

Here $\mathrm{V}_{\mathrm{O}} \approx 1.5 \times 10^{-23} \mathrm{~cm}^{3}$ is the volume per oxygen site in $\mathrm{r}-\mathrm{TiO}_{2}$.

Within our model, the grand potential contains the complete thermodynamic information of the problem. The local particle concentrations (particles per volume) $n_{k}(\vec{r})$ are obtained, up to a sign change, as the functional derivative of the grand potential with respect to a local variation of the respective chemical potential, namely

$$
n_{k}(\vec{r})=-\frac{\delta \Omega}{\delta \mu_{k}(\vec{r})}=\frac{1}{\mathrm{~V}_{\mathrm{O}}} \sum_{\sigma} P_{\sigma}(\vec{r}) \eta_{k, \sigma}
$$

for $k \in\{\mathrm{H}, \mathrm{O}\}$. For the electrons we need to add the contribution of the free charge carriers:

$$
\begin{aligned}
n_{e}(\vec{r})=-\frac{\delta \Omega}{\delta \mu_{e}(\vec{r})}= & \frac{1}{\mathrm{~V}_{\mathrm{O}}} \sum_{\sigma} P_{\sigma}(\vec{r}) \eta_{e, \sigma}+\left(\frac{m_{e} k_{\mathrm{B}} T}{2 \pi \hbar^{2}}\right)^{\frac{3}{2}} \\
& \times\left[N_{c}\left(\frac{m_{c}^{*}}{m_{e}}\right)^{\frac{3}{2}} e^{-\beta\left(\epsilon_{c}+v_{e}(\vec{r})-\mu_{e}\right)}\right. \\
& \left.-N_{v}\left(\frac{m_{v}^{*}}{m_{e}}\right)^{\frac{3}{2}} e^{-\beta\left(\mu_{e}-\epsilon_{v}-v_{e}(\vec{r})\right)}\right]
\end{aligned}
$$

The probability $P_{\sigma}(\vec{r})$ that an oxygen site at position $\vec{r}$ is in state $\sigma$, is, according to Eqs. (C5) and (C6),

$$
P_{\sigma}(\vec{r})=\frac{\exp \left(-\frac{1}{k_{\mathrm{B}} T}\left(E_{\sigma}+\eta_{e, \sigma} v_{e}(\vec{r})-\sum_{k} \eta_{k} \mu_{k}\right)\right)}{\sum_{\sigma=1}^{4} \exp \left(-\frac{1}{k_{\mathrm{B}} T}\left(E_{\sigma}+\eta_{e, \sigma} v_{e}(\vec{r})-\sum_{k} \eta_{k} \mu_{k}\right)\right)} .
$$

The charge density is $\rho(\vec{r})=-e n_{e}(\vec{r})$. The electric potential $\Phi(\vec{r})$ is connected with the potential acting on the electrons by $v_{e}(\vec{r})=-e \Phi(\vec{r})$. The Poisson equation closes the system of self-consistent equations

$$
\vec{\nabla}^{2} \Phi(\vec{r})=-\frac{1}{\epsilon_{0} \epsilon_{r}} \rho(\vec{r}) .
$$

The relative dielectric constant of $\epsilon_{r}=86$ has been taken from Parker [92].
For the description of the interface, the electric potential is allowed to vary only perpendicular to the interface. The distance from the interface is denoted by $z$, with the positive direction pointing towards the oxide. With $\vec{r}_{I}$, we denote a position at the interface and $\vec{r}_{B}$ is a position deep in the bulk oxide. The potential at the interface is thus $v_{e}\left(\vec{r}_{I}\right)=v_{e}(z=0)$ and that in the bulk is $v_{e}\left(\vec{r}_{B}\right)=v_{e}(z=+\infty)$.

The alignment of the band structures at the interface is determined by the Schottky-barrier height $\Phi_{\mathrm{Bn}}=\epsilon_{c}+v\left(\vec{r}_{I}\right)-$ $\mu_{e}$, defined as the difference of the conduction-band edge $\epsilon_{c}+v_{e}\left(\vec{r}_{I}\right)$ at the interface from the Fermi level $\mu_{e}$ of the metal. We have chosen its value $\Phi_{\mathrm{Bn}}=0.79 \mathrm{eV}[93,94]$ from the Schottky model as the metal work function minus the electron affinity of the oxide. Note that the interface between metal and oxides is affected by the local chemistry and the Schottky barrier. The Schottky barrier height alters for different terminations and has the dominant influence on the stability of impurities and dopants at the interface rather than the local chemistry.

In the oxide far from the interface, the position of the electron chemical potential $\mu_{e}$ relative to the band edges is determined by the charge-neutrality condition. Thus, the electric potential $v_{e}\left(\vec{r}_{B}\right)$ far from the interface relative to the electron chemical potential is a bulk property, which, however, depends on the chemical potentials of oxygen and hydrogen.

Near the interface, charges build up in the oxide, which produce an electric-potential step that aligns the chargeneutrality level of the bulk oxide with the Fermi level of the metal. The global charge neutrality is satisfied by a neutralizing charge buildup in the metal right at the interface. The charge density of the interface system can be expressed as

$$
\rho(z)=-e\left[\theta(z) n_{e}(z)-\delta\left(z-0^{-}\right) \int_{0^{+}}^{\infty} d z n_{e}(\vec{r})\right]
$$

with the the step function $\theta(z)$ and the delta function $\delta(z)$. This charge density defines the electron potential via the Poisson equation:

$$
\begin{aligned}
v_{e}(z)= & \mu_{e}+\frac{e}{\epsilon_{0} \epsilon_{r}} \delta\left(z-0^{-}\right)\left[z\left(\int_{0^{+}}^{\infty} d z^{\prime} n_{e}\left(z^{\prime}\right)\right)\right. \\
& \left.-\int_{0}^{z} d z \int_{0}^{z^{\prime}} d z^{\prime \prime} n_{e}\left(z^{\prime \prime}\right)\right] .
\end{aligned}
$$

Equations (C7), (C8), and (C11) form a coupled set of equations, that needs to be solved self-consistently.

In the self-consistent calculation of the potential profile has not been required, because the potential step $\Delta v_{e}$ between the interface and the bulk could be obtained from the Schottky barrier.

This concludes the description of the TMD model. The parameters used in the model are summarized in Tables III and $\mathrm{V}$.

\section{APPENDIX D: PARTIAL PRESSURES AND CHEMICAL POTENTIALS}

In order to link the TMD model to measurable quantities, we need to convert the partial pressures of the gases $\mathrm{O}_{2}$ and $\mathrm{H}_{2}$ in the environment into chemical potentials for the atoms. The resulting chemical potentials for oxygen and hydrogen 
TABLE V. Parameters used for the model of defect concentrations.

\begin{tabular}{lccc}
\hline \hline Effective electron mass & $m_{c}^{*}$ & $m_{e}$ & Ref. [91] \\
Effective hole mass & $m_{v}^{*}$ & $3.33 m_{e}$ & Ref. [91] \\
Conduction band multiplicity & $N_{c}$ & 4 & \\
Valence band multiplicity & $N_{v}$ & 2 & \\
Volume per oxygen site $\left(\mathrm{cm}^{3}\right)$ & $V_{\mathrm{O}}$ & $1.5 \times 10^{-23}$ & \\
Relative dielectric constant & $\epsilon_{r}$ & 86 & Ref. [92] \\
PBE band-gap (eV) & $\epsilon_{c}-\epsilon_{v}$ & 1.90 & \\
Schottky barrier $(\mathrm{eV})$ & $\Phi_{\mathrm{Bn}}$ & 0.79 & Ref. [61] \\
$k_{\mathrm{B}} T \ln \left[N_{c} \mathrm{~V}_{\mathrm{O}}\left(m_{c}^{*} k_{\mathrm{B}} T /\left(2 \pi \hbar^{2}\right)\right)^{\frac{3}{2}}\right]$ & & $-1.62 \mathrm{eV}$ & \\
\hline \hline
\end{tabular}

obtained for the individual processing steps are summarized in Table IV.

For dimeric molecules, the chemical potentials $\mu_{Y}$ for the atomic species, $Y \in\{\mathrm{O}, \mathrm{H}\}$, are obtained from the Gibbs free energy $G_{Y_{2}}(T, p, N)$ of the corresponding gas $Y_{2}$ at the specified partial pressure $p$ and temperature $T$. The chemical potentials $\mu_{Y_{2}}=G_{Y_{2}} / N_{Y_{2}}$ for the molecules are obtained as the Gibbs free energy per molecule. The chemical potential of an atom $\mu_{Y}=\frac{1}{2} \mu_{Y_{2}}$ is one-half of that of the dimeric molecule. Thus, we obtain

$$
\mu_{Y}=\frac{1}{2 N} G_{Y_{2}}(T, p, N) .
$$

The molecular Gibbs free energy (dropping the subscript $Y_{2}$ )

$$
\begin{aligned}
G(T, p, N)= & N E_{\mathrm{tot}}+G_{\mathrm{trans}}(T, p, N)+F_{\mathrm{vib}}(T, N) \\
& +F_{\mathrm{rot}}(T, N)+F_{e}(T, N)
\end{aligned}
$$

is divided into the total energy $E_{\text {tot }}$ of the molecules without their zero-point energy, its contribution $G_{\text {trans }}$ from molecular translations, the free energy $F_{\text {vib }}$ of internal vibration including the zero-point energy, the free energy $F_{\text {rot }}$ of the molecular rotations, as well as the contribution from spin multiplicity,
TABLE VI. Molecular parameters [95] used to convert temperatures and partial pressures via Eq. (D1) into chemical potentials.

\begin{tabular}{cccc}
\hline \hline & $g_{e}$ & $d$ & $\hbar \omega /(2 \pi \hbar c)$ \\
\hline $\mathrm{O}_{2}$ & 3 & $1.208 \AA$ & $1580.19 \mathrm{~cm}^{-1}$ \\
$\mathrm{H}_{2}$ & 1 & $0.741 \AA$ & $4163.17 \mathrm{~cm}^{-1}$ \\
\hline
\end{tabular}

The translational contribution to the Gibbs potential

$$
G_{\text {trans }}(T, p, N)=N k_{\mathrm{B}} T \ln \left(\frac{\lambda_{T}^{3} p}{k_{\mathrm{B}} T}\right)
$$

depends on the thermal de Broglie wave length $\lambda_{T}=\sqrt{\frac{2 \pi \hbar^{2}}{M k_{\mathrm{B}} T}}$, where $M$ is the mass of the molecule. As we consider a dilute gas of molecules, the interaction between molecules is ignored, so that we use the Gibbs free energy of the ideal gas.

The vibrational free energy is expressed by the vibrational frequency $\omega$ as

$$
F_{\text {vib }}(T, N)=\frac{N}{2} \hbar \omega+N k_{B} T \ln \left[1-\exp \left(-\frac{\hbar \omega}{k_{B} T}\right)\right] .
$$

It contains the zero-point energy as well as the quantum mechanical entropy term.

The rotational part is obtained

$$
F_{\text {rot }}(T, N)=-N k_{B} T \ln \left[\sum_{\ell=0}^{\infty}(2 \ell+1) \mathrm{e}^{-\frac{\hbar^{2} \ell(\ell+2)}{\mu d^{2} k_{B} T}}\right] .
$$

The effective mass $\mu$ of the dimer molecule is one-half of the atomic masses, respectively one-fourth of the molecular mass $M$, i.e., $\mu=\frac{1}{4} M$. The bond distance is denoted as $d$.

For the oxygen molecule also the spin multiplicity $g_{e}=3$ contributes to the free energy in the form

$$
F_{e}(T, N)=-N k_{B} T \ln \left(g_{e}\right) .
$$

The molecular parameters entering Eq. (D1) are summarized in Table VI.
[1] U. Diebold, Surf. Sci. Rep. 48, 53 (2003).

[2] U. Diebold, S.-C. Li, and M. Schmid, Annu. Rev. Phys. Chem. 61, 129 (2010).

[3] S. J. Tauster, S. C. Fung, R. T. K. Baker, and J. A. Horsley, Science 211, 1121 (1981).

[4] U. Roland, T. Braunschweig, and F. Roessner, J. Mol. Catal. A: Chem. 127, 61 (1997).

[5] J. Y. Park, L. R. Baker, and G. A. Somorjai, Chem. Rev. 115, 2781 (2015).

[6] M. K. Nowotny, L. R. Sheppard, T. Bak, and J. Nowotny, J. Phys. Chem. C 112, 5275 (2008).

[7] M. Ni, M. K. H. Leung, D. Y. C. Leung, and K. Sumathy, Renew. Sust. Energ. Rev. 11, 401 (2007).

[8] R. Waser, R. Dittmann, G. Staikov, and K. Szot, Adv. Mater. 21, 2632 (2009).

[9] Y. Ma, X. Wang, Y. Jia, X. Chen, H. Han, and C. Li, Chem. Rev. 114, 9987 (2014).
[10] M. Bowker, P. Stone, R. Bennett, and N. Perkins, Surf. Sci. 497, 155 (2002)

[11] J. Sá, J. Bernardi, and J. A. Anderson, Catal. Lett. 114, 91 (2007).

[12] J. J. Liu, ChemCatChem 3, 934 (2011).

[13] X. Chen, L. Liu, and F. Huang, Chem. Soc. Rev. 44, 1861 (2015).

[14] O. Ola and M. M. Maroto-Valer, J. Photochem. Photobiol., C: Photochem. Rev. 24, 16 (2015).

[15] V. Burlaka, V. Roddatis, M. D. Bongers, and A. Pundt, Sci. Rep. 7, 9564 (2017).

[16] V. Roddatis, M. D. Bongers, R. Vink, V. Burlaka, J. Č́́žek, and A. Pundt, J. Phys. Chem. Lett. 9, 5246 (2018).

[17] B. Mohammad, M. A. Jaoude, V. Kumar, D. M. Al Homouz, H. A. Nahla, M. Al-Qutayri, and N. Christoforou, Nanotechnol. Rev. 5, 311 (2016).

[18] A. Moballegh and E. C. Dickey, Acta Mater. 86, 352 (2015). 
[19] D.-H. Kwon, K. M. Kim, J. H. Jang, J. M. Jeon, M. H. Lee, G. H. Kim, X.-S. Li, G.-S. Park, B. Lee, S. Han et al., Nat. Nanotechnol. 5, 148 (2010).

[20] H. Kobayashi, K. Kishimoto, and Y. Nakato, Surf. sci. 306, 393 (1994).

[21] M. Cerchez, H. Langer, M. E. Achhab, T. Heinzel, D. Ostermann, H. Lüder, and J. Degenhardt, Appl. Phys. Lett. 103, 033522 (2013).

[22] M. Strungaru, M. Cerchez, S. Herbertz, T. Heinzel, M. E. Achhab, and K. Schierbaum, Appl. Phys. Lett. 106, 143109 (2015).

[23] U. Roland, R. Salzer, T. Braunschweig, F. Roessner, and H. Winkler, J. Chem. Soc., Faraday Trans. 91, 1091 (1995).

[24] F. A. Grant, Rev. Mod. Phys. 31, 646 (1959).

[25] M. K. Nowotny, T. Bak, and J. Nowotny, J. Phys. Chem. B 110, 16270 (2006).

[26] M. K. Nowotny, T. Bak, and J. Nowotny, J. Phys. Chem. B 110, 16283 (2006).

[27] M. K. Nowotny, T. Bak, and J. Nowotny, J. Phys. Chem. B 110, 16292 (2006).

[28] M. K. Nowotny, T. Bak, and J. Nowotny, J. Phys. Chem. B 110, 16302 (2006).

[29] J. Nowotny, J. Phys. Chem. C 115, 18316 (2011).

[30] T. Bak, J. Nowotny, M. Rekas, and C. C. Sorrell, J. Phys. Chem. Solids 64, 1043 (2003).

[31] T. Bak, J. Nowotny, M. Rekas, and C. C. Sorrell, J. Phys. Chem. Solids 64, 1057 (2003).

[32] T. Bak, J. Nowotny, M. Rekas, and C. C. Sorrell, J. Phys. Chem. Solids 64, 1069 (2003).

[33] O. W. Johnson, J. DeFord, and J. W. Shaner, J. Appl. Phys. 44, 3008 (1973).

[34] P. W. Peacock and J. Robertson, Appl. Phys. Lett. 83, 2025 (2003).

[35] F. Filippone, G. Mattioli, P. Alippi, and A. Amore Bonapasta, Phys. Rev. B 80, 245203 (2009).

[36] F. Herklotz, E. V. Lavrov, and J. Weber, Phys. Rev. B 83, 235202 (2011).

[37] T. S. Bjørheim, A. Kuwabara, and T. Norby, J. Phys. Chem. C 117, 5919 (2013).

[38] U. Aschauer and A. Selloni, Phys. Chem. Chem. Phys. 14, 16595 (2012).

[39] M. Sotoudeh, S. J. Hashemifar, M. Abbasnejad, and M. R. Mohammadizadeh, AIP Adv. 4, 027129 (2014).

[40] M. Sotoudeh, M. Abbasnejad, and M. R. Mohammadizadeh, Eur. Phys. J. Appl. Phys. 67, 30401 (2014).

[41] L. B. Mo, Y. Wang, Y. Bai, Q. Y. Xiang, Q. Li, W. Q. Yao, J. O. Wang, K. Ibrahim, H. H. Wang, C. H. Wan, and J. L. Cao, Sci. Rep. 5, 17634 (2015).

[42] P. B. Wells and A. G. Wilkinson, Top. Catal. 5, 39 (1998).

[43] E. Wicke, H. Brodowsky, and H. Züchner, Hydrogen in palladium and palladium alloys, in Hydrogen in Metals II: Application-Oriented Properties, edited by G. Alefeld and J. Völkl (Springer, Berlin, 1978), pp. 73-155.

[44] U. Muschiol, P. K. Schmidt, and K. Christmann, Surf. Sci. 395, 182 (1998).

[45] D. Farías, P. Schilbe, M. Patting, and K.-H. Rieder, J. Chem. Phys. 110, 559 (1999).

[46] D. Teschner, J. Borsodi, A. Wootsch, Z. Révay, M. Hävecker, A. Knop-Gericke, S. D. Jackson, and R. Schlögl, Science 320, 86 (2008).
[47] B. Brandt, W. Ludwig, J.-H. Fischer, J. Libuda, F. Zaera, and S. Schauermann, J. Catal. 265, 191 (2009).

[48] G. Sandrock, S. Suda, L. Schlapbach, and L. Schlapbach, Top. Appl. Phys. 67, 197 (1992).

[49] F. D. Manchester, A. San-Martin, and J. M. Pitre, J. Phase Equilib. 15, 62 (1994).

[50] A. Sieverts, Zeitschrift für Metallkunde 21, 37 (1929).

[51] T. B. Flanagan and W. A. Oates, Annu. Rev. Mater. Sci. 21, 269 (1991).

[52] A. Pundt and R. Kirchheim, Annu. Rev. Mater. Res. 36, 555 (2006).

[53] R. Kirchheim, Acta Mater. 55, 5129 (2007).

[54] R. Kirchheim, Acta Mater. 55, 5139 (2007).

[55] S. Wagner and A. Pundt, Int. J. Hydrog. Energy 41, 2727 (2016).

[56] S. Wagner, T. Kramer, H. Uchida, P. Dobron, J. Cizek, and A. Pundt, Acta Mater. 114, 116 (2016).

[57] H. Mehrer, Diffusion in Solids: Fundamentals, Methods, Materials, Diffusion-Controlled Processes (Springer Science \& Business Media, New York, 2007), Vol. 155.

[58] W. Mönch, Electronic Properties of Semiconductor Interfaces (Springer Science \& Business Media, New York, 2004), Vol. 43.

[59] R. T. Tung, Appl. Phys. Rev. 1, 011304 (2014).

[60] M. Paulose, O. K. Varghese, G. K. Mor, C. A. Grimes, and K. G. Ong, Nanotechnology 17, 398 (2005).

[61] M. D. Bongers, In situ studies on palladium/rutile titanium dioxide exposed to low pressure hydrogen gas environments, Ph.D. thesis, Niedersächsische Staats- und Universitätsbibliothek Göttingen, Georg-August-Universität Göttingen (2018).

[62] M. J. Süess, E. Mueller, and R. Wepf, Ultramicroscopy 111, 1224 (2011).

[63] R. Krause-Rehberg and H. S. Leipner, Positron Annihilation in Semiconductors: Defect Studies (Springer-Verlag, Berlin, 1999).

[64] P. Hohenberg and W. Kohn, Phys. Rev. 136, B864 (1964).

[65] W. Kohn and L. J. Sham, Phys. Rev. 140, A1133 (1965).

[66] J. P. Perdew, K. Burke, and M. Ernzerhof, Phys. Rev. Lett. 77, 3865 (1996).

[67] P. E. Blöchl, Phys. Rev. B 50, 17953 (1994).

[68] CP-PAW website, http://www2.pt.tu-clausthal.de/paw/ (revision 1178).

[69] P. E. Blöchl and C. Först, arXiv:1210.5937.

[70] O. Jepson and O. K. Anderson, Solid State Commun. 9, 1763 (1971).

[71] G. Lehmann and M. Taut, Phys. Stat. Sol. (b) 54, 469 (1972).

[72] P. E. Blöchl, O. Jepsen, and O. K. Andersen, Phys. Rev. B 49, 16223 (1994).

[73] D. T. Cromer and K. Herrington, J. Am. Chem. Soc. 77, 4708 (1955).

[74] E. Stoyanov, F. Langenhorst, and G. Steinle-Neumann, Am. Mineral. 92, 577 (2007).

[75] R. Brydson, H. Sauer, W. Engel, J. M. Thomass, E. Zeitler, N. Kosugi, and H. Kuroda, J. Phys.: Condens. Matter 1, 797 (1989).

[76] S. O. Kucheyev, T. van Buuren, T. F. Baumann, J. H. Satcher, T. M. Willey, R. W. Meulenberg, T. E. Felter, J. F. Poco, S. A. Gammon, and L. J. Terminello, Phys. Rev. B 69, 245102 (2004).

[77] J. Pascual, J. Camassel, and H. Mathieu, Phys. Rev. B 18, 5606 (1978). 
[78] S.-D. Mo and W. Y. Ching, Phys. Rev. B 51, 13023 (1995).

[79] L. D. Finkelstein, E. Z. Kurmaev, M. A. Korotin, A. Moewes, B. Schneider, S. M. Butorin, J.-H. Guo, J. Nordgren, D. Hartmann, M. Neumann, and D. L. Ederer, Phys. Rev. B 60, 2212 (1999).

[80] W. F. Kuhs and M. S. Lehmann, J. Phys. Chem. 87, 4312 (1983).

[81] B. Klose and H. Klose, Die Erdatmosphre: Ihre chemische Zusammensetzung, vertikale Struktur und Physik, Meteorologie: Eine interdisziplinäre einführung in die physik der atmosphäre (Springer, Berlin, 2015), pp. 15-84.

[82] R. Haul and G. Dümbgen, J. Phys. Chem. Solids 26, 1 (1965).

[83] M. Arita, M. Hosoya, M. Kobayashi, and M. Someno, J. Am. Ceram. Soc. 62, 443 (1979).

[84] D. J. Derry, D. G. Lees, and J. M. Calvert, J. Phys. Chem. Solids 42, 57 (1981).

[85] www.clue.physik.uni-goettingen.de.

[86] P. J. Schultz and K. G. Lynn, Rev. Mod. Phys. 60, 701 (1988).
[87] W. Anwand, G. Brauer, M. Butterling, H. R. Kissener, and A. Wagner, Defect and Diffusion Forum 331, 25 (2012).

[88] M. Heidelmann, J. Barthel, and L. Houben, Ultramicroscopy 109, 1447 (2009).

[89] K. Sader, B. Schaffer, G. Vaughan, R. Brydson, A. Brown, and A. Bleloch, Ultramicroscopy 110, 998 (2010).

[90] D. B. Williams and C. B. Carter, Transmission Electron Microscopy, 2nd ed. (Springer US, New York, 2009).

[91] T. V. Perevalov and V. A. Gritsenko, J. Exp. Theor. Phys. 112, 310 (2011).

[92] R. A. Parker, Phys. Rev. 124, 1719 (1961).

[93] L. A. DuBridge and W. W. Roehr, Phys. Rev. 39, 99 (1932).

[94] A. Imanishi, E. Tsuji, and Y. Nakato, J. Phys. Chem. C 111, 2128 (2007).

[95] T. Shimanouchi, Tables of Molecular Vibrational Frequencies. Consolidated Volume I (National Bureau of Standards, Washington, DC, 1972). 